\title{
Real-Time Dynamic Spectrum Management for Multi-User Multi-Carrier Communication Systems
}

\author{
Paschalis Tsiaflakis, Member, IEEE, François Glineur, and Marc Moonen, Fellow, IEEE
}

\begin{abstract}
Dynamic spectrum management is recognized as a key technique to tackle interference in multi-user multi-carrier communication systems and networks. However existing dynamic spectrum management algorithms may not be suitable when the available computation time and compute power are limited, i.e., when a very fast responsiveness is required. In this paper, we present a new paradigm, theory and algorithm for realtime dynamic spectrum management (RT-DSM). Specifically, a RT-DSM algorithm is real-time in the sense that it can be stopped at any point in time while guaranteeing a feasible and improved solution. This is enabled by the introduction of a novel difference-of-variables (DoV) transformation and problem reformulation, for which a primal coordinate ascent approach is proposed with exact line search via a logarithmically-scaled grid search. The proposed algorithm is referred to as iterative power difference balancing (IPDB). Simulations for different realistic wireline and wireless interference-limited systems demonstrate its good performance, low complexity and wide applicability under different configurations.
\end{abstract}

Index Terms-Dynamic spectrum management, interference management, multi-user, multi-carrier, real-time.

\section{INTRODUCTION}

$\mathbf{I}$ NTERFERENCE is a key performance-limiting factor in many state-of-the-art communication systems and networks [1]-[7]. In particular, when multiple users transmit simultaneously in a common frequency bandwidth, significant interference levels can be observed among them in practical systems. This can result in large data rate reductions [6]-[8], poor spectral and energy efficiency [9]-[15], unstable behaviour

Manuscript received July 25, 2013; revised November 15, 2013 and January 18,2014 . The editor coordinating the review of this paper and approving it for publication was $\mathrm{G}$. Colavolpe.

P. Tsiaflakis was funded by the Research Foundation-Flanders (FWO). This research work was carried out in the frame of KU Leuven Research Council CoE PFV/10/002 (OPTEC), KU Leuven Bilateral Scientific Cooperation Project Tsinghua University 2012-2014, the Belgian Programme on Interuniversity Attraction Poles IUAP P7/23 BESTCOM 2012-2017 and Phase VII/19 DYSCO 2012-2017, Concerted Research Action GOA-MaNet, Research Project FWO nr. G.091213, and IWT Project CONGA. The scientific responsibility is assumed by its authors.

P. Tsiaflakis is with the Department of Electrical Engineering (ESAT), STADIUS Center for Dynamical Systems, Signal Processing and Data Analytics, KU Leuven, Kasteelpark Arenberg 10 bus 2446, B-3001 Leuven, Belgium, and with Bell Labs, Alcatel-Lucent, Copernicuslaan 50, B-2018 Antwerp, Belgium (e-mail: Paschalis.Tsiaflakis@alcatel-lucent.com).

F. Glineur is with the Center for Operations Research and Econometrics, Université Catholique de Louvain, B-1348 Louvain-la-Neuve, Belgium, and with the Institute of Information and Communication Technologies, Electronics and Applied Mathematics, Université Catholique de Louvain, B-1348 Louvain-la-Neuve, Belgium (e-mail: Francois.Glineur@uclouvain.be).

Marc Moonen is with the Department of Electrical Engineering (ESAT), STADIUS Center for Dynamical Systems, Signal Processing and Data Analytics, KU Leuven, Kasteelpark Arenberg 10 bus 2446, B-3001 Leuven, Belgium (e-mail: Marc.Moonen@esat.kuleuven.be).

Digital Object Identifier 10.1109/TCOMM.2014.012614.130580 due to transient interference [16]-[19], unfairness due to unbalanced interference impact [20] and other performance degradations.

Dynamic spectrum management (DSM) is recognized as an important technique to tackle these performance degradations in such interference-limited systems [5]-[8], [10]-[42]. In digital subscriber line (DSL) literature, DSM is typically categorized into three levels, namely DSM 1, DSM 2 and DSM 3. DSM 1 corresponds to single-user management in terms of impulse-noise control, delay parameter tuning and transmit spectrum shaping [43]. DSM 2 addresses solutions where the transmit spectra of all users are jointly managed [5], [7], [26] so as to prevent the destructive impact of interference. DSM 3 is also referred to as vectoring and consists of the application of signal coordination methods that can actively cancel interference between users [6]-[8], [24], [25]. We briefly highlight here that the word 'dynamic' in DSM does not refer to time dynamic adaptation of the spectrum management resources, but rather to the adaptation of the spectrum management resources in order to take the concrete physical channel conditions of the considered scenario into account.

In this paper the focus is on DSM through the management of transmit spectra for general multi-user multi-carrier systems, including wireline DSL systems (corresponding to DSM 2) as well as wireless interference-limited systems. Here, the transmit spectra of all users in the system are jointly managed and optimized, where each user employs a multicarrier transmission technique such as orthogonal frequency division multiplexing (OFDM) or discrete multitone (DMT). In the remainder of this paper, we will refer to this technique shortly by DSM, as this term is similarly used in other literature [7], [26], [29]. Furthermore we follow a standard interference channel system model where the interference is treated as additive white Gaussian (AWG) noise, which is a very common practical model in operational networks [7].

Research work on DSM has progressed significantly over the last decade. More specifically, a whole range of DSM algorithms has been proposed ranging from centralized [30][35], to distributed [21], [41], [42], [44], [45] and autonomous algorithms [21], [36]-[40]. Each of these has its specific properties in terms of computational complexity and level of coordination. We refer to [21], [26] and references therein for an overview and a comparison of DSM algorithms proposed in DSL literature. The DSL setting represents one relevant example of a multi-user multi-carrier interference channel. However DSM is also of interest in several wireless settings, where similar algorithms have been proposed. Examples are 
multicell downlink DSM or inter-cell interference coordination for heterogeneous networks [37], DSM for multi-user multichannel cellular relay networks [44], and OFDM-based cognitive radio systems [9], [46].

However, none of the previously proposed DSM algorithms have addressed real-time computation constraints. More specifically, when computation time and compute power are limited, there is no guarantee that a suitable solution can be found with existing DSM algorithms. This is because existing DSM algorithms typically follow an iterative approach where it is not known in advance how many iterations are required to converge to a feasible and sufficiently accurate solution. Furthermore, existing DSM algorithms typically follow a dual decomposition approach where solution feasibility and good performance is not guaranteed until after convergence. In addition, an important issue when tackling the nonconvex DSM problem in the dual domain is the possible non-zero duality gap, due to the finite number of subcarriers used in the multi-carrier transmission [28], [29].

Our focus in this paper is to tackle the above issues by a new paradigm and theory of real-time dynamic spectrum management. The corresponding real-time dynamic spectrum management algorithms succeed in working under real-time constraints where the computation time and the compute power are limited. This property is highly desirable when real-time responsiveness to changes in the network is to be guaranteed, such as varying channel and noise characteristics, users joining or leaving the network, changing QoS requirements, crosslayer control, etc. To the best of our knowledge, no literature on resource allocation for interference-limited communication systems addresses such real-time constraints.

To enable this new paradigm, we first propose a novel transformation, referred to as the difference-of-variables (DoV) transformation, which transforms the standard DSM problem into a problem with alternative primal variables, referred to as power difference variables. With this reformulation in hand, a first real-time DSM algorithm is proposed, which is referred to as iterative power difference balancing (IPDB). This algorithm combines the DoV problem reformulation with a solution that follows a coordinate ascent approach with exact line search via a logarithmically-scaled grid search. The combination of these two ingredients results in an efficient algorithm for which the effectiveness and real-time behaviour are analyzed and evaluated for different settings.

The paper is organized as follows. Section II briefly describes the multi-user multi-carrier system model and DSM. Section III first gives a definition of real-time DSM, then introduces the DoV transformation and problem. Finally, the IPDB algorithm is presented. This is extended with a procedure for dealing with inequality power constraints and equalization. The performance for different wireline and wireless scenarios and settings is presented in Section IV.

\section{SYSTEM MODEL AND DYNAMIC SPECTRUM MANAGEMENT}

We consider a multi-user communication system with a set $\mathcal{N}=\{1, \ldots, N\}$ of $N$ communication links over a common frequency band. Each link consists of a transmitter-receiver pair, and is also referred to as a user. In addition, each user employs a multi-carrier transmission scheme, such as OFDM or DMT. We assume perfect synchronization and a cyclic prefix length longer than the maximum channel length (considering direct as well as interference channels), so that the user data are transmitted independently and in parallel on the different subcarriers, also referred to as tones. The set of $K$ tones is denoted as $\mathcal{K}=\{1, \ldots, K\}$. All users can transmit on all tones, resulting in overlapping transmit spectra and thus multi-user interference. Note that our system also includes the single-user case, i.e., with $N=1$, as a special case.

We focus on dynamic spectrum management through multiuser multi-carrier transmit power management and optimization. No signal coordination or vectoring between transmitters or receivers is assumed. Each user thus employs a single-user decoder. This case is well in line with many practical settings where a distinct physical location or a limited communication between transmitters and receivers does not allow for signal coordination. We follow the common standard interference channel system model where the multi-user interference is treated as AWG noise. Perfect channel state information is assumed at transmitters and receivers. The achievable bit rate of user $n$ on tone $k$ is then given as follows

$$
b_{k}^{n}\left(\mathbf{s}_{k}\right) \triangleq \log _{2}\left(1+\frac{s_{k}^{n}}{\sum_{m \neq n} a_{k}^{n, m} s_{k}^{m}+z_{k}^{n}}\right),
$$

with $s_{k}^{n}$ denoting the transmit power of user $n$ on tone $k$, $\mathbf{s}_{k}=\left[s_{k}^{1}, \ldots, s_{k}^{N}\right]^{T}, a_{k}^{n, m}$ denoting the normalized channel gain from user $m$ to user $n$ on tone $k$, and $z_{k}^{n}$ denoting the normalized received noise power for user $n$ on tone $k$. We note that normalization corresponds to dividing by the respective direct channel gain of user $n$ and tone $k$. A signal-to-noise ratio (SNR) gap [47] that characterizes imperfect coding and signal modulation, and a noise margin, may be included in the normalized channel gains and noise power.

The DSM problem can then be formulated as follows

$$
\begin{array}{cl}
\underset{s_{k}^{n}, k \in \mathcal{K}, n \in \mathcal{N}}{\operatorname{maximize}} & \sum_{n \in \mathcal{N}} w_{n} R^{n}\left(\mathbf{s}_{k}, k \in \mathcal{K}\right) \\
\text { subject to } & P^{n}\left(s_{k}^{n}, k \in \mathcal{K}\right)=P^{n, \text { tot }}, \forall n \in \mathcal{N} \\
& 0 \leq s_{k}^{n} \leq s_{k}^{n, \text { mask }}, \forall n \in \mathcal{N}, \forall k \in \mathcal{K}, \\
\text { with } & \left\{\begin{array}{l}
P^{n}\left(s_{k}^{n}, k \in \mathcal{K}\right) \triangleq \sum_{k \in \mathcal{K}} s_{k}^{n}, \\
R^{n}\left(\mathbf{s}_{k}, k \in \mathcal{K}\right) \triangleq \sum_{k \in \mathcal{K}} b_{k}^{n}\left(\mathbf{s}_{k}\right)
\end{array}\right.
\end{array}
$$

with $R^{n}\left(\mathbf{s}_{k}, k \in \mathcal{K}\right)$ denoting the achievable data rate for user $n$ and its corresponding weighting $w_{n}, P^{n}\left(s_{k}^{n}, k \in \mathcal{K}\right)$ denoting the total allocated (transmit) power of user $n$, the constant $P^{n \text {,tot }}$ denoting the total power budget for user $n$, and the constant $s_{k}^{n \text {,mask }}$ denoting the maximum transmit power (spectral mask) of user $n$ on tone $k$. This corresponds to a maximization of the sum of the weighted achievable data rates (with multiple tones), under per-user total power constraints and per-tone spectral masks.

The transmit spectrum of a user refers to the user's transmit power on all tones. These transmit spectra are the optimization 
variables for the DSM problem.

Observe that the per-user total power constraints are expressed as equality constraints. This will be extended to inequality constraints in Section III-D.

\section{Real-Time Dynamic Spectrum Management}

Real-time computation is an important challenge in practice where computation time and compute power of communication devices and systems are limited. In this section, we present a new paradigm and theory for real-time dynamic spectrum management (RT-DSM). We first introduce our definition of a RT-DSM algorithm in Section III-A. To enable the design of RT-DSM algorithms, we then propose a novel transformation, also referred to as a difference-of-variables transformation, in Section III-B. Using this transformation, we reformulate the DSM problem in terms of power difference variables. This allows for the design of a first RT-DSM algorithm in Section III-C, referred to as iterative power difference balancing. This is further extended with a procedure for dealing with per-user total power inequality constraints in Section III-D, and an equalization procedure to tackle nonsmooth solution behaviour in Section III-E.

\section{A. Definition of Real-Time Dynamic Spectrum Management Algorithm}

To provide a concrete label and definition of the algorithms targeted in this paper, we introduce the following definition:

Definition 1: [Real-time dynamic spectrum management (RT-DSM) algorithm] A real-time dynamic spectrum management algorithm sequentially updates the transmit power variables such that these satisfy all constraints after each update.

This definition implies that RT-DSM algorithms can be stopped after each update (even after a single update), and as such they are suitable for execution under very tight computation time and compute power budgets, as they can be stopped whenever one of both resources is exhausted. This guarantees fast responsiveness, and allows for real-time operation.

\section{B. Difference-of-Variables Transformation and Optimization}

The original DSM problem (2) consists of a separable objective function and coupled per-user total power constraints. An important step towards the design of RT-DSM algorithms is to eliminate the coupling per-user total power constraints. To enable this, we propose to replace the primal variables $s_{k}^{n} \forall n, k$ with an alternative set of primal variables $t_{k}^{n} \forall n, k$, where the latter will be referred to as the power difference variables. For this we propose a novel transformation of variables, referred to as the difference-of-variables (DoV) transformation:

$$
\begin{aligned}
& s_{k}^{n}=\sum_{j \in \mathcal{K}} \beta_{k}^{n}(j) t_{j}^{n}+P^{n, \text { tot }} \gamma_{k}^{n}, \quad n \in \mathcal{N}, k \in \mathcal{K}(4) \\
\text { with } & \sum_{k \in \mathcal{K}} \beta_{k}^{n}(j)=0, \quad n \in \mathcal{N}, j \in \mathcal{K} \\
& \sum_{k \in \mathcal{K}} \gamma_{k}^{n}=1, \quad n \in \mathcal{N} \\
& \beta_{k}^{n}(k)>0, \quad n \in \mathcal{N}, k \in \mathcal{K}
\end{aligned}
$$

where $\beta_{k}^{n}(j), \gamma_{k}^{n}$ are (fixed) arbitrary constants that can take any value satisfying constraints (5), (6) and (7). We also define the following set $\mathcal{B}_{k}^{n}$ for later use

$$
\mathcal{B}_{k}^{n}=\left\{j \in \mathcal{K} \mid \beta_{k}^{n}(j) \neq 0\right\},
$$

which denotes the set of tones for user $n$ and tone $k$ with power difference variables that influence $s_{k}^{n}$.

Using the DoV transformation (4), we obtain a reformulation of (2) as given in the following theorem:

Theorem 1: Applying a DoV transformation (4), satisfying constraints (5) (6) (7), to the DSM problem (2) results in the equivalent reformulated problem (3).

Proof: The objective and constraints of (3) can be obtained by applying the DoV transformation to the objective and per-tone spectral mask constraints of (2). The per-user total power constraints of (2) are not present anymore in the reformulation (3). This is because the proposed DoV transformation (4) ensures that these constraints are satisfied for all values of the power difference variables $t_{k}^{n}$. This can straightforwardly be proven as follows:

$$
\begin{aligned}
\sum_{k \in \mathcal{K}} s_{k}^{n} & =\sum_{k \in \mathcal{K}}\left(\sum_{j \in \mathcal{K}} \beta_{k}^{n}(j) t_{j}^{n}+P^{n, \text { tot }} \gamma_{k}^{n}\right) \\
& =(\sum_{j \in \mathcal{K}} t_{j}^{n} \underbrace{\sum_{k \in \mathcal{K}} \beta_{k}^{n}(j)}_{=0})+(P^{n, \text { tot }} \underbrace{\sum_{k \in \mathcal{K}} \gamma_{k}^{n}}_{=1}) \\
& =P^{n, \text { tot }}
\end{aligned}
$$

The strength of reformulation (3) is that the coupled peruser total power constraints are eliminated, and that the reformulation is expressed in terms of the power difference variables $t_{k}^{n}$ only. Because of the constraint (5), each power difference variable $t_{k}^{n}$ adds some transmit power to some tones but subtracts the same amount of transmit power from other tones, resulting in a zero total power change operation. This is also the reason why $t_{k}^{n}$ is referred to as a power difference variable. The above properties of the reformulated DSM problem (3) are crucial to enable the design of RT-DSM algorithms, as will be shown in Section III-C.

Reformulation (3) displays coupling in both the objective and the constraints. However, the coupling can be of much smaller size (compared to (2)) in the sense that it couples only a subset of all tones. More specifically, the bit loading $b_{k}^{n}$ on a given tone and the spectral mask constraints are impacted by a number of power difference variables equal to the cardinality of $\mathcal{B}_{k}^{n}$, which can be as low as two. In contrast in (2), $s_{k}^{n}$ 


$$
\begin{array}{ll}
\underset{t_{k}^{n}, k \in \mathcal{K}, n \in \mathcal{N}}{\operatorname{maximize}} & \sum_{n \in \mathcal{N}} w_{n} \sum_{k \in \mathcal{K}} \log _{2}\left(1+\frac{\sum_{j \in \mathcal{K}} \beta_{k}^{n}(j) t_{j}^{n}+P^{n, \text { tot }} \gamma_{k}^{n}}{\sum_{m \neq n} a_{k}^{n, m}\left(\sum_{j \in \mathcal{K}} \beta_{k}^{m}(j) t_{j}^{m}+P^{m, \text { tot }} \gamma_{k}^{m}\right)+z_{k}^{n}}\right) \\
\text { subject to } & 0 \leq \sum_{j \in \mathcal{K}} \beta_{k}^{n}(j) t_{j}^{n}+P^{n, \text { tot }} \gamma_{k}^{n} \leq s_{k}^{n \text {,mask }}, \forall n \in \mathcal{N}, \forall k \in \mathcal{K}
\end{array}
$$

$$
\begin{aligned}
& \underset{t_{k}^{n}}{\operatorname{maximize}} f_{k}^{n}\left(t_{k}^{n}\right) \\
& \text { subject to } \quad t_{k}^{n, \min } \leq t_{k}^{n} \leq t_{k}^{n, \max } \\
& \left(f_{k}^{n}\left(t_{k}^{n}\right)=\sum_{k \in \mathcal{A}_{k}^{n}} \sum_{n=1}^{N} w_{n} \log _{2}\left(1+\frac{\sum_{j \in \mathcal{B}_{k}^{n}} \beta_{k}^{n}(j) t_{j}^{n}+P^{n, \text { tot }} \gamma_{k}^{n}}{\sum_{m \neq n} a_{k}^{n, m}\left(\sum_{j \in \mathcal{B}_{k}^{m}} \beta_{k}^{m}(j) t_{j}^{m}+P^{m, \text { tot }} \gamma_{k}^{m}\right)+z_{k}^{n}}\right)\right. \\
& \text { with } \\
& \left\{\begin{array}{l}
x=\max \left\{-\frac{1}{\beta_{q}^{n}(k)}\left(\sum_{j \in \mathcal{B}_{q}^{n} \backslash\{k\}} \beta_{q}^{n}(j) t_{j}^{n}+P^{n, \text { tot }} \gamma_{q}^{n}\right), \forall q \in \mathcal{A}_{k}^{n} \& \beta_{q}^{n}(k)>0\right\} \\
y=\max \left\{\frac{1}{\beta_{q}^{n}(k)}\left(s_{q}^{n, \text { mask }}-\left(\sum_{j \in \mathcal{B}_{q}^{n} \backslash\{k\}} \beta_{q}^{n}(j) t_{j}^{n}+P^{n, \text { tot }} \gamma_{q}^{n}\right)\right), \forall q \in \mathcal{A}_{k}^{n} \& \beta_{q}^{n}(k)<0\right\} \\
t_{k}^{n, \min }=\max \{x, y\} \\
u=\min \left\{\frac{1}{\beta_{q}^{n}(k)}\left(s_{q}^{n, \text { mask }}-\left(\sum_{j \in \mathcal{B}_{q}^{n} \backslash\{k\}} \beta_{q}^{n}(j) t_{j}^{n}+P^{n, \text { tot }} \gamma_{q}^{n}\right)\right), \forall q \in \mathcal{A}_{k}^{n} \& \beta_{q}^{n}(k)>0\right\} \\
v=\min \left\{-\frac{1}{\beta_{q}^{n}(k)}\left(\sum_{j \in \mathcal{B}_{q}^{n} \backslash\{k\}} \beta_{q}^{n}(j) t_{j}^{n}+P^{n, \text { tot }} \gamma_{q}^{n}\right), \forall q \in \mathcal{A}_{k}^{n} \& \beta_{q}^{n}(k)<0\right\} \\
t_{k}^{n, \max }=\min \{u, v\}
\end{array}\right.
\end{aligned}
$$

impacts all terms because of the coupled per-user total power constraints.

Two examples of valid DoV transformations, i.e., satisfying (5) (6) (7), are as follows:

1) Two-tone DoV transformation:

$$
s_{k}^{n}=\left\{\begin{array}{l}
t_{k}^{n}-t_{k-1}^{n}+P^{n, \text { tot }} \gamma_{k}^{n}, k>1 \\
t_{1}^{n}-t_{K}^{n}+P^{n, \text { tot }} \gamma_{1}^{n}, k=1
\end{array}\right.
$$

2) Three-tone DoV transformation:

$$
s_{k}^{n}=\left\{\begin{array}{l}
-t_{k+1}^{n}+2 t_{k}^{n}-t_{k-1}^{n}+P^{n, \text { tot }} \gamma_{k}^{n}, 1<k<N \\
-t_{2}^{n}+2 t_{1}^{n}-t_{K}^{n}+P^{n, \text { tot }} \gamma_{1}^{n} \quad, k=1 \\
-t_{1}^{n}+2 t_{K}^{n}-t_{K-1}^{n}+P^{n, \text { tot }} \gamma_{K}^{n} \quad, k=N
\end{array}\right.
$$

Note that $\gamma_{k}^{n}$ is still arbitrary here, for example $\gamma_{k}^{n}=$ $1 / K, \forall k \in \mathcal{K}$. The two-tone DoV transformation has a coupling over two consecutive tones, i.e., $\operatorname{card}\left(\mathcal{B}_{k}^{n}\right)=2$, whereas for the three-tone DoV transformation $\operatorname{card}\left(\mathcal{B}_{k}^{n}\right)=3$.

\section{Iterative Power Difference Balancing}

Our RT-DSM algorithm design starts from the proposed reformulated problem (3). As the DSM problem corresponds to an NP-hard nonconvex problem [29], we propose an iterative coordinate ascent approach to tackle it, which we refer to as iterative power difference balancing. More specifically, it consists in sequentially updating/optimizing one power difference variable at a time. The corresponding one-dimensional optimization problem is given in (9), where the only optimization variable is the single power difference variable $t_{k}^{n}$.
To identify the coupling level, we define a set $\mathcal{A}_{k}^{n}$ as follows,

$$
\mathcal{A}_{k}^{n}=\left\{j \in \mathcal{K} \mid \beta_{j}^{n}(k) \neq 0\right\}
$$

which denotes the set of tones for user $n$ and tone $k$ whose transmit powers are influenced by power difference variable $t_{k}^{n}$. The objective function in (9) is coupled over multiple tones, depending on the cardinality of $\mathcal{A}_{k}^{n}$. However, a proper choice of the DoV transformation results in a small coupling level, reducing the sum to only a few terms. For instance, for the two-tone DoV transformation (10), this corresponds to two terms, which means that we only consider two tones in the objective function and the constraints. The constraints in problem (9) correspond to plain bound constraints, where the bounds $t_{k}^{n, \min }$ and $t_{k}^{n, \max }$ are simple constants that depend on the value of the other power difference variables, which are kept constant in the considered iteration. By updating the power difference variables one at a time, the total power $P^{n}$ is not changed because of the zero per-user total power change property (5). Each update results in an improved objective function value, guaranteeing a monotonously improving performance. We stress that, in contrast to typical existing DSM algorithms, IPDB solves the problem in the primal domain instead of the dual domain, avoiding all issues related to a possible non-zero duality gap.

The one-dimensional problem (9) however still corresponds to a nonconvex problem, and therefore we propose to solve it with a plain one-dimensional (1D) exhaustive search, where the interval $\left[t_{k}^{n, \min }, t_{k}^{n, \max }\right]$ is discretized in small steps. This can be seen as an exact line search based on a 1D grid search. Note that iterative grid-based exhaustive one- 
dimensional searches have been shown to be very promising in DSM literature, such as for the iterative spectrum balancing (ISB) algorithm [31], [32]. We emphasize however that these existing algorithms focus on dual solutions where the discretization is applied to the primal variables, which are transmit powers. In our case, we focus on a primal solution where we consider power difference variables. As a result, we claim that we can use a coarser discretization, because power difference variables focus on differences between tones. Taking into account the fact that the channels (direct as well as crosstalk channels) vary over tones with some degree of smoothness, the optimal transmit spectra do not differ significantly from one tone to the next, a property that has also been referred to as spectral correlation [48]. Therefore we propose to use a fine discretization for small difference values and a coarse discretization for large differences. More specifically we choose a logarithmically-scaled discretization. To obtain this we define the following sets

$$
\begin{aligned}
\mathcal{F}^{+} & =\left\{x \mid 10 \log _{10}(x)=-140+k \delta, k \in \mathbb{N}\right\} \\
\mathcal{F} & =\mathcal{F}^{+} \cup\{0\} \cup\left(-\mathcal{F}^{+}\right) \\
\mathcal{J}_{k}^{n} & =\mathcal{F} \cap\left[t_{k}^{n, \text { min }}, t_{k}^{n, \text { max }}\right],
\end{aligned}
$$

where $\delta$ is a discretization variable (referred to as granularity), which defines the relative power difference accuracy. The chosen value of -140 for set $\mathcal{F}^{+}$corresponds to the minimum non-zero power difference in $\mathrm{dBm} / \mathrm{Hz}$. We chose a value of $-140 \mathrm{dBm} / \mathrm{Hz}$ to guarantee a good accuracy and smooth behaviour even for power levels in the 120 to $140 \mathrm{dBm} / \mathrm{Hz}$ range. However, a larger value can be chosen depending on the desired accuracy. Note that in the case of dual algorithms such as ISB, one typically chooses an absolute power level discretization with a granularity of $0.5 \mathrm{dBm} / \mathrm{Hz}$. However for IPDB, we show in Section IV-B that a coarser granularity can be chosen (e.g., $\delta=1 \mathrm{dBm} / \mathrm{Hz}$ ) without significantly impacting the final accuracy, which then significantly reduces computational complexity. The zero element is included in the set $\mathcal{F}$ to maintain monotonicity.

The resulting grid-based search approach for the 1D problem corresponds to the following problem (13), where the feasible space consists of set $\mathcal{J}_{k}^{n}$ :

$$
\operatorname{maximize}_{t_{k}^{n} \in \mathcal{J}_{k}^{n}} f_{k}^{n}\left(t_{k}^{n}\right) \text {. }
$$

The full IPDB algorithm is given in Algorithm 1. In line 1, the power difference variables and the granularity $\delta$ are initialized. In line 2, the constants $\gamma_{k}^{n}$ are initialized satisfying two different constraints. A straightforward choice here is $\gamma_{k}^{n}=1 / K$, which corresponds to an equal power allocation over all tones, i.e., $s_{k}^{n}=P^{n \text {,tot }} / K$, and typically satisfies all power constraints in (2) and (3). The loop in line 3 repeats until some stopping criterion is achieved, or until some real-time deadline is reached. Line 4 is the per-user loop. Note that the user order is not defined and can be arbitrarily chosen. In fact this user order can also include multiple instances of the same user. Line 5 is the inner per-user iteration with a maximum of $I$ iterations. Line 6 is the per-tone loop. Again, the tone order is not necessarily consecutive but can be arbitrary. Line 7 is the only line that involves an update of the transmit powers and corresponds to a one-dimensional power difference variable update by a 1D exhaustive grid-based search of problem (13) over the values $\mathcal{J}_{k}^{n}$. With the DoV transformation (4) the corresponding updated transmit powers can be obtained. In line 9 the power difference variables are then reset and the constants $\gamma_{k}^{n}$ are updated so as to keep the transmit powers fixed. Although the latter two actions are not necessary from a theoretical point of view, they are seen to improve the performance, as the values around $t_{k}^{n}=0$ are discretized at a finer granularity. This can be seen as a recentering operation so as to fully benefit from the logarithmically-scaled discretization. As a result a fine granularity in transmit powers can be obtained through a sum of coarse power difference variables (that are not coarse everywhere). This results in a good final solution accuracy as demonstrated in Section IV. Lines 12 and 13 correspond to optional procedures to consider inequalities and to perform equalization, as discussed in Section III-D and III-E, respectively. Note however that these steps are not necessary and can be disregarded at this point.

We now analyze different aspects and properties of the IPDB algorithm:

1) Tunability: We want to highlight that the IPDB algorithm offers flexibility in choosing the user order, the tone order, the number of inner iterations, the granularity $\delta$, and in the initialization of the parameters $\gamma_{k}^{n}$. Different choices are evaluated in Section IV-A.

2) Real-time Property: The IPDB algorithm satisfies the real-time property from Definition III-A: it can be stopped at any moment as it satisfies the constraints after every single update of the power difference variables, which have a one-to-one mapping to the transmit powers through (4). The concrete improved real-time behaviour is demonstrated in Section IV-A4.

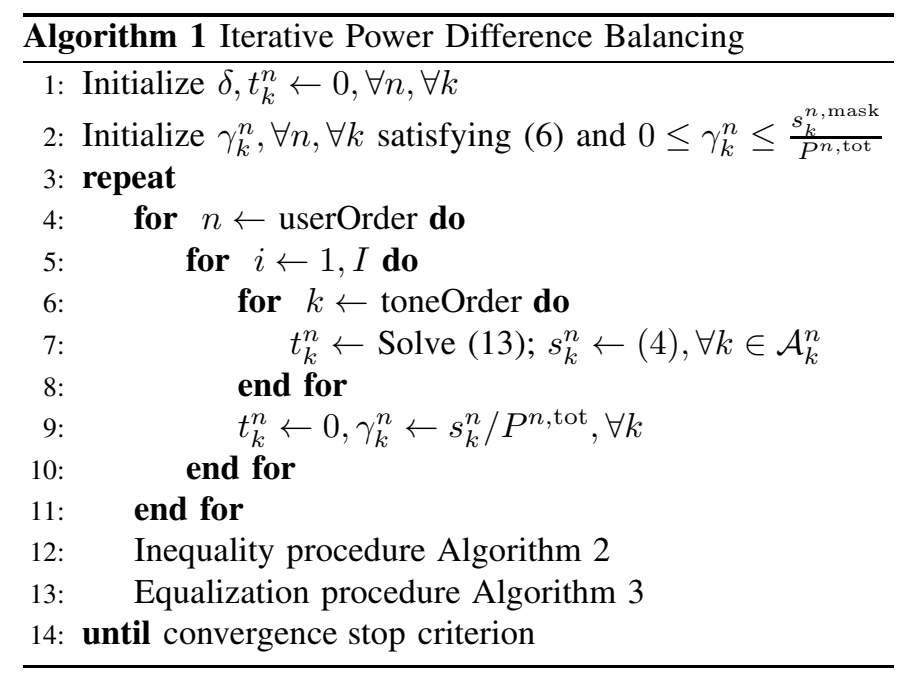

3) Complexity: The computational complexity analysis of IPDB is rather straightforward. Most of the complexity results from line 7, which corresponds to a simple 1D exhaustive grid-based search. The total computational complexity thus roughly corresponds to the number of $1 \mathrm{D}$ exhaustive gridbased searches until convergence. Under a given computational and compute power budget, it is easy to determine the number of updates that can be performed, which demonstrates the benefit of the real-time property of IPDB. 
4) Monotonicity: Each update results in a non-decreasing feasible objective function value. As a result IPDB has an interesting monotonicity and scalability property, where more computation time or compute power consistently results in a better obtained solution.

5) Convergence: As the IPDB algorithm is a coordinate search method, the convergence behaviour is inherited from such methods. The looser the coupling between the coordinate ascent variables, the faster the convergence [49]. Guaranteed convergence to a stationary point is difficult to claim theoretically. Under the hypothesis that each coordinate search optimum is unique, a standard theorem guarantees convergence to a stationary point. More precisely, Proposition 2.7.1 in [50] states that when optimizing a continuously differentiable function over a feasible region defined as a Cartesian product of closed convex sets (each set corresponding to a disjoint block of variables), every limit point of the sequence of iterates of the (block)coordinate-descent method is a stationary point ${ }^{1}$ provided the optimum computed at each iteration is uniquely attained. This is not necessarily always the case for our 1D problem. However, plain convergence of IPDB (not necessarily to a stationary point but) to a final objective function value can be guaranteed, as each coordinate search optimizes the objective function along that direction. It is important to highlight that because of the real-time property that ensures constraint satisfaction after each single update, and the monotonicity property that ensures a non-decreasing objective function value, it is not extremely important that full convergence is reached when performing the IPDB algorithm. Fast numerical convergence results (up to $99 \%$ and $99.9 \%$ of full performance convergence) are demonstrated in Section IV-A.

Finally, we want to highlight that although we employ a DoV transformation with differences between the transmit powers on different tones for one user, one could in principle also employ differences between the transmit powers of different users on a single tone or different tones if there are per-tone sum power constraints or total network sum power constraints, respectively.

\section{Inequality constraints}

In this section we consider inequality constraints for the peruser total power constraints as given by the following DSM problem

$$
\begin{array}{ll}
\underset{s_{k}^{n}, k \in \mathcal{K}, n \in \mathcal{N}}{\operatorname{maximize}} & \sum_{n \in \mathcal{N}} w_{n} R^{n}\left(\mathbf{s}_{k}, k \in \mathcal{K}\right) \\
\text { subject to } & P^{n}\left(s_{k}^{n}, k \in \mathcal{K}\right) \leq P^{n, \text { tot }}, \forall n \in \mathcal{N} \\
& 0 \leq s_{k}^{n} \leq s_{k}^{n, \text { mask }}, \forall n \in \mathcal{N}, \forall k \in \mathcal{K} .
\end{array}
$$

To extend IPDB to also allow inequality constraints, we propose an inequality procedure that allows to reduce the per-user total powers below $P^{n \text {,tot }}$, whenever this improves the weighted sum of achievable data rates. This procedure is given in Algorithm 2. Let $\alpha>1$ and $0<\beta<1$. At

\footnotetext{
${ }^{1}$ Actually an errata available at http://www.athenasc.com/nlperrate.pdf adds a very mild additional monotonicity condition.
}

line $2 s_{\alpha}$ computes a value larger than the current value $s_{k}^{n}$ while satisfying the per-user total power constraint as well as the spectral mask constraint. Then at line $3 s_{\beta}$ computes a value smaller than the current value $s_{k}^{n}$. In line 4 , a per-tone weighted sum of bit rates evaluation for user $n$ is performed to check if the weighted sum of bit rates can be improved by increasing or decreasing the transmit power $s_{k}^{n}$. (notation: $\left.\mathbf{s}_{k}\right|_{s_{k}^{n}=\hat{s}_{k}^{n}}$ is meant to designate $\mathbf{s}_{k}$ with $s_{k}^{n}$ replaced by $\hat{s}_{k}^{n}$.) This is repeated for all tones. This inequality procedure does not violate the real-time property as the constraints of (14) are satisfied after every single update. Similarly the monotonicity property is not violated, as each update results in a nondecreasing feasible objective function value.

\begin{tabular}{ll}
\hline Algorithm 2 Inequality procedure user $n$ \\
\hline 1: for $\quad k \leftarrow$ toneOrder do \\
$\begin{array}{ll}\text { 2: } & s_{\alpha} \leftarrow \min \left(\alpha s_{k}^{n}, P^{n, \text { tot }}-\sum_{q \in \mathcal{K} \backslash k} s_{q}^{n}, s_{k}^{n, \text { mask }}\right) \\
\text { 3: } & s_{\beta} \leftarrow \beta s_{k}^{n} \\
\text { 4: } & s_{k}^{n} \leftarrow \underset{\hat{s}_{k}^{n} \in\left\{s_{\alpha}, s_{k}^{n}, s_{\beta}\right\}}{\operatorname{argmax}} \sum_{m \in \mathcal{N}} w_{m} b_{k}^{m}\left(\mathbf{s}_{k} \mid s_{k}^{n}=\hat{s}_{k}^{n}\right) \\
\text { 5: } & \text { end for }\end{array}$ \\
\hline
\end{tabular}

\section{E. Randomization and Equalization}

As mentioned in Section III-C, the IPDB algorithm is tunable in the tone order, the user order, and in the initial choice of $\gamma_{k}^{n}$, where the latter has a one-to-one mapping with the initial transmit powers if the power difference variables $t_{k}^{n}$ are fixed. We can use randomized values, i.e., a random tone order, random user order and random initial transmit powers. Randomization in iteration orders and initial conditions has been shown to be effective in several cases in literature [51]. It is shown in Section IV that randomization indeed results in performance gains.

However, randomization also has some side effects which may not always be desirable. For instance, when randomizing the initial transmit powers, the resulting transmit spectra may display a very non-smooth behaviour, in the sense that transmit powers differ significantly from one tone to the next. For instance, in Figure 1 the resulting transmit spectra are shown when applying IPDB to a 2-user scenario (corresponding to the blue and the green curves) with randomized initial transmit spectra. The transmit spectra between tones 45 and 115 display significant jumps. The reason is that by starting from very different initial transmit powers on consecutive tones, IPDB can converge to very different per-tone solutions in consecutive tones. We want to highlight that the non-smooth behaviour results in a typically better overall performance and prevents convergence to a very poor solution in which a poor choice is systematically made over multiple consecutive tones.

However, such non-smooth solution behaviour is not always desirable in practice. For instance, with current (legacy) DSL modems there are restrictions on the shape of practical transmit powers. One such restriction is that a user's transmit spectrum is configured by a limited number $(\leq 32)$ of transmit spectrum shaping (tssi) breakpoints that consist of a tone index and the associated (discretized) transmit power value. 


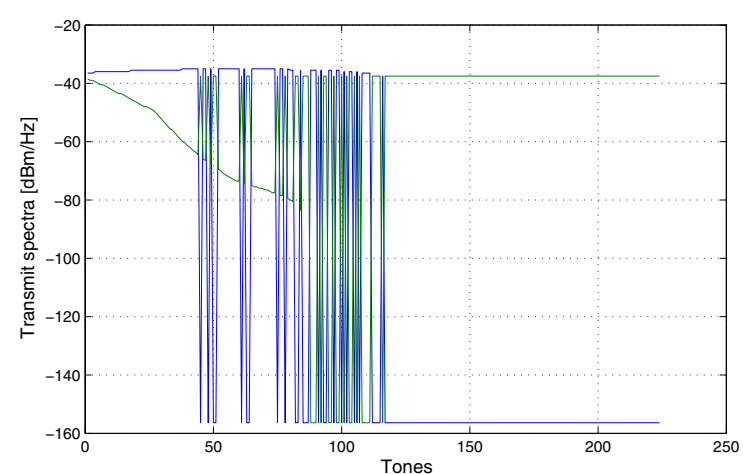

Fig. 1. Resulting transmit powers for 2-user ADSL scenario of Figure 2 when using IPDB with randomized initial transmit spectra and without equalization. The transmit spectra between tones 45 and 115 display significant jumps.

This set of breakpoints has to comply with a number of restrictions where for instance the (inband) transmit power from one tone to another tone cannot vary more than 0.75 $\mathrm{dB}$ [52]. Non-smooth transmit powers over tones are thus not desired. Therefore we propose an equalization procedure to obtain smoother transmit powers. This procedure is given in Algorithm 3 and consists of one simple for loop over the tones. In each loop, it is checked if a spike can be detected between three (almost) consecutive tones $k, k+1$ and $k+3$. If a down spike is detected (with more than $10 \mathrm{~dB}$ difference), this is averaged out. If an up spike is detected (with more than $10 \mathrm{~dB}$ difference) this is flattened out. The reason tone $k+3$ instead of tone $k+2$ is considered for the third tone is that in this way one-tone wide spikes as well as two-tone wide spikes can be detected. Although the above procedure is a very low-complexity operation, it has very good equalization performance resulting in smooth transmit spectra, as demonstrated in Section IV. From an optimization point of view, the equalization procedure can be seen as a procedure that allows to obtain overall solutions where similar per-tone solutions are chosen in neighboring tones.

Moreover this equalization procedure does not have to be called in all iterations but only after every so many outer iterations.

The equalization procedure can slightly violate the real-time property during execution of line 10 and lines 12 to 14 , but as these steps are of much smaller granularity than the main computational step of IPDB, i.e. line 7 of Algorithm 1, the real-time property is essentially preserved.

Finally, although the monotonicity property may be violated whenever the equalization procedure is called, this seems to provide a better overall performance as demonstrated in Section IV.

\section{Simulations and Performance Analysis}

In this section, the performance of the IPDB algorithm is evaluated for different settings and for different performance metrics. Performance will be compared with that of the popular ISB algorithm, which is also a coordinate ascent gridbased search algorithm. In contrast to IPDB which operates in the primal domain, ISB is based on a combination of a dual decomposition approach with a discrete per-tone coordinate

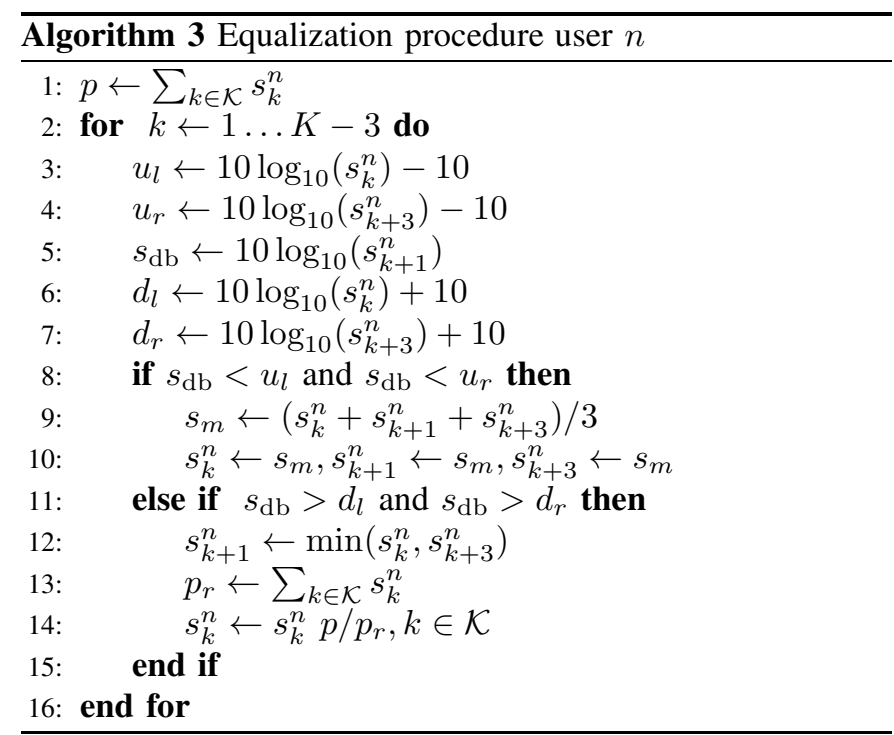

ascent grid-based search. We note that ISB does not satisfy the real-time property, and is thus not an RT-DSM algorithm. As ISB is a well-established DSL DSM algorithm with good performance and low complexity, we think that an empirical validation of both algorithms is a fair and relevant comparison to demonstrate the merits of the proposed method for the DSL DSM application field. IPDB will also be compared with two standard algorithms for nonconvex continuous optimization: a trust-region method and an interior-point method. Next to wireline DSL settings, wireless LTE settings will also be considered.

For the wireline DSL simulations in Sections IV-A IV-B IV-C, we use realistic DSL simulators, which have been validated in practice and are aligned with standards. We consider 24 AWG twisted copper pair lines. The maximum transmit power is $20.4 \mathrm{dBm}$ for the ADSL and ADSL2+ scenarios, and $11.5 \mathrm{dBm}$ for the VDSL scenarios. The SNR gap is chosen at $12.9 \mathrm{~dB}$ for the DSL scenarios, corresponding to a coding gain of $3 \mathrm{~dB}$, a noise margin of $6 \mathrm{~dB}$, and a target symbol error probability of $10^{-7}$. The tone spacing is $4.3125 \mathrm{kHz}$. The DMT symbol rate is $4 \mathrm{kHz}$. The weights $w_{n}$ are chosen equal for all users $(n=1 \ldots N)$, namely $w_{n}=1 / N$, unless specified otherwise. When the equalization procedure is activated in Algorithm 1, it is only performed after each fifth outer iteration.

A concrete wireless LTE heterogeneous network setting will be discussed in Section IV-D.

\section{A. IPDB performance: ADSL Case}

The ADSL scenario under consideration is the near-far scenario shown in Figure 2, i.e., a 2-user downstream scenario with one far-user connected to the central office $(\mathrm{CO})$ and a second near-user connected to a remote terminal (RT). This near-far type of scenarios is quite common in practice and has received a lot of attention in DSL literature. The underlying optimization problem is known to display a very nonconvex behaviour for which locally optimal DSM methods can perform poorly [21]. In our simulations, the RT-connected user is given a weight of 0.1 and the $\mathrm{CO}$-connected user a 


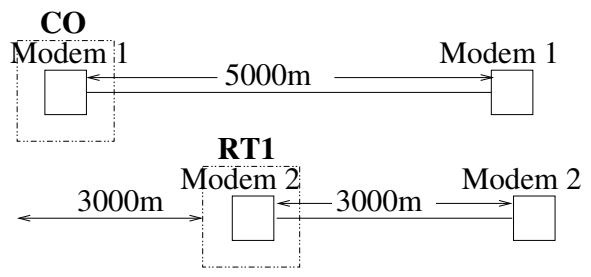

Fig. 2. Near-far 2-user ADSL downstream scenario with central office (CO) and remote terminal (RT).

weight of 0.9 , to prevent the latter being allocated a too small achievable data rate.

The performance of both IPDB and ISB are evaluated for different configurations exploiting the tunability of IPDB. The tone order corresponds to one configuration setting for which we test four choices. TO 1 and TO 2 correspond to tone orders $[1: K]$ and $[K:-1: 1]$, respectively. TO 3 selects TO 1 or TO 2 with probability of $50 \%$ each, each time line 6 of IPDB is entered. TO 4 corresponds to a tone order which is a fully random permutation of the tone set $[1: K]$, and which changes each time line 6 of IPDB is entered.

A second configuration setting corresponds to the initial transmit power spectra. For this we consider two choices: (1) EP corresponds to an equal power allocation, i.e., $\gamma_{k}^{n}=$ $1 / K \forall k, n$, (2) RP corresponds to a random power allocation with uniformly distributed probabilities in $\mathrm{dB}$ scale while satisfying (6) and $0 \leq \gamma_{k}^{n} \leq s_{k}^{n \text {,mask }} / P^{n \text {,tot }}$. The number of inner iterations $I$ is fixed at 1 .

We consider IPDB with and without equalization (Algorithm 3), corresponding to EQ ON and EQ OFF, respectively. Three different DoV transformations are evaluated, which correspond to (10), (15) and (16), respectively, and where $\pi$ is a vector that corresponds to a random permutation of vector $[1, \ldots, K]$ :

1) Two-tone rand DoV transformation:

$$
s_{k}^{n}=t_{k}^{n}-t_{\pi(k)}^{n}+P^{n, \text { tot }} \gamma_{k}^{n}
$$

2) Three-tone 2 DoV transformation:

$$
s_{k}^{n}= \begin{cases}2 t_{k}^{n}-t_{k+1}^{n}-t_{k+2}^{n}+P^{n, \text { tot }} \gamma_{k}^{n} & , k<N-2 \\ 2 t_{N-1}^{n}-t_{N}^{n}-t_{1}^{n}+P^{n, \text { tot }} \gamma_{N-1}^{n} & , k=N-1 \\ 2 t_{N}^{n}-t_{1}^{n}-t_{2}^{n}+P^{n, \text { tot }} \gamma_{N}^{n} \quad, k=N\end{cases}
$$

We also consider different granularities for the discrete searches, where we consider the standard $0.5 \mathrm{dBm} / \mathrm{Hz}$ [30], [31] for the transmit powers $s_{k}^{n}$ in ISB, and two coarser granularities of $1 \mathrm{dBm} / \mathrm{Hz}$ and $10 \mathrm{dBm} / \mathrm{Hz}$ for the power difference variables $t_{k}^{n}$ in IPDB.

Finally, for all configurations, the results are averaged over 15 different runs to obtain an averaged performance.

1) Weighted Sum of Achievable Data Rates Performance: In Table I the weighted sum of achievable data rates performance is compared for IPDB and ISB under the different configurations.

The standard ISB configuration corresponds to the settings TO 1, EQ OFF, EP and $0.5 \mathrm{dBm} / \mathrm{Hz}$ granularity. For this standard setting, ISB has a weighted sum of achievable data rates performance of $1.5549 \mathrm{Mbps}$. This increases spectacularly when applying randomized initial transmit powers, i.e., RP with up to $1.8274 \mathrm{Mbps}$. In addition, applying the equalization procedure (EQ ON) further improves the performance up to $1.8799 \mathrm{Mbps}$, when combined with RP. This is quite surprising as the equalization procedure not only provides smoother resulting transmit spectra (as shown in Section IV-A5) but in addition also improves the achievable data rate performance. The explanation is that in the case of randomization most of the tones converge to good per-tone solutions. When combining this with the equalization procedure, this will cause the fewer poor per-tone solutions to be forced to the larger set of good per-tone solutions, resulting in a better overall performance.

The performance of IPDB is good when using the two-tone rand DoV transformation (15). For a $1 \mathrm{~dB}$ granularity, one can see a performance improvement of up to $22 \%$ compared to the standard ISB configuration, and up to $1 \%$ compared to the best ISB configuration with RP and EQ ON. The IPDB performance is not so good for the two-tone and three-tone 2 DoV transformations (10) and (16), respectively. However when combining these DoV transformations with the equalization procedure, their performance is improved spectacularly. Reducing the granularity from $1 \mathrm{dBm} / \mathrm{Hz}$ steps to $10 \mathrm{dBm} / \mathrm{Hz}$ steps decreases the performance, for all cases. It is interesting to notice that the tone order does not play a significant role.

To summarize achievable data rate performance, the twotone rand DoV transformation with equalization activated and $1 \mathrm{dBm} / \mathrm{Hz}$ granularity offers the best performance, and performs better than all ISB configurations.

2) Convergence Speed: Tables II and III display the convergence speed in terms of the number of outer iterations (loop corresponding to lines 3-14) to converge to $99 \%$ and $99.9 \%$ weighted achievable sum data rate performance, respectively.

The number of outer iterations varies strongly for different IPDB configurations. A very fast convergence is achieved for the two-tone rand DoV transformation. In particular, we see convergence after less than 10 outer iterations (for $99 \%$ performance) and 16 outer iterations (for $99.9 \%$ performance), when using the settings EQ OFF and $1 \mathrm{dBm} / \mathrm{Hz}$ granularity. For the EQ ON setting, this increases to only 20 outer iterations for both $1 \mathrm{dBm} / \mathrm{Hz}$ and $10 \mathrm{dBm} / \mathrm{Hz}$ granularity. The other DoV transformations require up to 200 iterations to converge. In general, a larger granularity results in a slower convergence.

ISB only requires 2 to 6 outer iterations to converge. However, we stress that ISB requires a dual optimization step to satisfy the total power constraints within each outer iteration, which is not required for IPDB. The complexity per outer iteration is thus much larger for ISB.

For illustration, the evolution of the weighted sum of achievable data rates and the per-user total powers is displayed in Figures 3 and 4 as a function of the outer iterations for a single run. It can be seen that $99 \%$ and $99.9 \%$ performance are achieved after 8 and 12 outer iterations. The per-user total powers constraints are always satisfied, which demonstrates the real-time property of IPDB.

3) Complexity Comparison IPDB: To compare the relative computational complexity of IPDB and ISB, Tables IV and $\mathrm{V}$ display the relative number of bit rate calculations (1) for 
TABLE I

WEIGHTED SUM OF DATA RATES PERFORMANCE [MBPS] FOR THE NEAR-FAR CO-RT SCENARIO OF FIG. 2 WITH DIFFERENT SETTINGS FOR IPDB. METHOD TWO-TONE RAND USES DOV TRANSFORMATION (15). TONE ORDER (TO) $1=[1: \mathrm{K}]$, TONE ORDER $2=$ [K:- $1: 1]$, TONE ORDER $3=$ UNIFORM PROBABILITY OF 1 AND 2, TONE ORDER 4 = RANDOM PERMUATION, EP = CONSTANT EQUAL INIT POWER, RP = RANDOM INIT POWER SATISFYING TOTAL POWER CONSTRAINT. TWO RIGHT MOST COLUMNS CORRESPOND TO ISB PERFORMANCE.

\begin{tabular}{|c|c|c|c|c|c|c|c|c|c|c|c|c|c|c|c|}
\hline \multirow{3}{*}{\multicolumn{2}{|c|}{$\begin{array}{c}\text { Method } \\
\text { Equalization } \\
\text { Granularity }\end{array}$}} & \multicolumn{4}{|c|}{ Two-tone } & \multicolumn{4}{|c|}{ Two-tone rand } & \multicolumn{4}{|c|}{ Three-tone 2} & \multicolumn{2}{|c|}{ ISB } \\
\hline & & \multicolumn{2}{|c|}{ EQ OFF } & \multicolumn{2}{|c|}{ EQ ON } & \multicolumn{2}{|c|}{ EQ OFF } & \multicolumn{2}{|c|}{ EQ ON } & \multicolumn{2}{|c|}{ EQ OFF } & \multicolumn{2}{|c|}{ EQ ON } & \multirow{2}{*}{$\frac{\text { EQ OFF }}{0.5 \mathrm{~dB}}$} & \multirow{2}{*}{$\frac{\text { EQ ON }}{0.5 \mathrm{~dB}}$} \\
\hline & & $1 \mathrm{~dB}$ & $10 \mathrm{~dB}$ & $1 \mathrm{~dB}$ & $10 \mathrm{~dB}$ & $1 \mathrm{~dB}$ & $10 \mathrm{~dB}$ & $1 \mathrm{~dB}$ & $10 \mathrm{~dB}$ & $1 \mathrm{~dB}$ & $10 \mathrm{~dB}$ & $1 \mathrm{~dB}$ & $10 \mathrm{~dB}$ & & \\
\hline \multirow{2}{*}{ TO 1} & EP & 1.5080 & 1.4223 & 1.8608 & 1.6542 & 1.8542 & 1.8320 & 1.8978 & 1.8519 & 1.5143 & 1.3780 & 1.8324 & 1.3875 & 1.5549 & 1.5549 \\
\hline & $\mathrm{RP}$ & 1.3858 & 1.3739 & 1.7538 & 1.7892 & 1.8087 & 1.8247 & 1.8951 & 1.8775 & 1.4065 & 1.3930 & 1.8135 & 1.7490 & 1.8274 & 1.8799 \\
\hline \multirow{2}{*}{ TO 2} & EP & 1.5826 & 1.4626 & 1.7914 & 1.6759 & 1.7872 & 1.8358 & 1.8761 & 1.8374 & 1.5066 & 1.3552 & 1.8439 & 1.3298 & & \\
\hline & $\mathrm{RP}$ & 1.3724 & 1.3388 & 1.7069 & 1.7689 & 1.7940 & 1.8212 & 1.8806 & 1.8507 & 1.4114 & 1.3657 & 1.7985 & 1.7705 & & \\
\hline \multirow{2}{*}{ TO 3} & EP & 1.5393 & 1.4459 & 1.8647 & 1.6432 & 1.8201 & 1.8345 & 1.8731 & 1.8611 & 1.4978 & 1.3772 & 1.8316 & 1.3758 & & \\
\hline & $\mathrm{RP}$ & 1.3957 & 1.3834 & 1.7759 & 1.7380 & 1.7975 & 1.8236 & 1.8831 & 1.8503 & 1.3887 & 1.4134 & 1.8428 & 1.7149 & & \\
\hline \multirow{2}{*}{ TO 4} & EP & 1.4428 & 1.4422 & 1.8543 & 1.5426 & 1.8240 & 1.8308 & 1.8739 & 1.8394 & 1.4564 & 1.3624 & 1.7925 & 1.4202 & & \\
\hline & $\mathrm{RP}$ & 1.3972 & 1.3948 & 1.8045 & 1.7071 & 1.8099 & 1.8147 & 1.8854 & 1.8620 & 1.4265 & 1.3709 & 1.8375 & 1.7113 & & \\
\hline
\end{tabular}

TABLE II

CONVERGENCE SPEED [NB OF OUTER ITERATIONS] TO CONVERGE TO 99\% PERFORMANCE FOR NEAR-FAR CO-RT SCENARIO OF FIG. 2 WITH DIFFERENT SETTINGS FOR IPDB. METHOD TWO-TONE RAND IS WITH PERMUTATED TONES ON THE TRANSFORMATION. TONE ORDER (TO) $1=[1: \mathrm{K}]$, TONE ORDER $2=$ [K:-1:1], TONE ORDER $3=$ UNIFORM PROBABILITY OF 1 AND 2, TONE ORDER $4=$ RANDOM PERMUATION, EP $=$ CONSTANT EQUAL INIT POWER, RP = RANDOM INIT POWER SATISFYING TOTAL POWER CONSTRAINT. TWO RIGHT MOST COLUMNS CORRESPOND TO ISB PERFORMANCE.

\begin{tabular}{|c|c|c|c|c|c|c|c|c|c|c|c|c|c|c|c|}
\hline \multirow{3}{*}{\multicolumn{2}{|c|}{$\begin{array}{c}\text { Method } \\
\text { Equalization } \\
\text { Granularity }\end{array}$}} & \multicolumn{4}{|c|}{ Two-tone } & \multicolumn{4}{|c|}{ Two-tone rand } & \multicolumn{4}{|c|}{ Three-tone 2} & \multicolumn{2}{|c|}{ ISB } \\
\hline & & \multicolumn{2}{|c|}{ EQ OFF } & \multicolumn{2}{|c|}{ EQ ON } & \multicolumn{2}{|c|}{ EQ OFF } & \multicolumn{2}{|c|}{ EQ ON } & \multicolumn{2}{|c|}{ EQ OFF } & \multicolumn{2}{|c|}{ EQ ON } & \multirow{2}{*}{$\frac{\text { EQ OFF }}{0.5 \mathrm{~dB}}$} & \multirow{2}{*}{$\frac{E Q \mathrm{ON}}{0.5 \mathrm{~dB}}$} \\
\hline & & $1 \mathrm{~dB}$ & $10 \mathrm{~dB}$ & $1 \mathrm{~dB}$ & $10 \mathrm{~dB}$ & $1 \mathrm{~dB}$ & $10 \mathrm{~dB}$ & $1 \mathrm{~dB}$ & $10 \mathrm{~dB}$ & $1 \mathrm{~dB}$ & $10 \mathrm{~dB}$ & $1 \mathrm{~dB}$ & $10 \mathrm{~dB}$ & & \\
\hline \multirow{2}{*}{ TO 1} & EP & 46.00 & 40.00 & 150.00 & 162.00 & 5.67 & 8.20 & 10.53 & 19.67 & 80.00 & 56.00 & 101.00 & 103.00 & 2.00 & 2.00 \\
\hline & $\mathrm{RP}$ & 41.33 & 61.67 & 124.13 & 162.13 & 8.60 & 14.60 & 17.87 & 15.00 & 31.67 & 44.13 & 133.20 & 169.00 & 2.00 & 5.00 \\
\hline \multirow{2}{*}{ TO 2} & EP & 29.00 & 52.00 & 61.00 & 174.00 & 5.20 & 8.00 & 10.87 & 8.73 & 22.00 & 59.00 & 116.00 & 177.00 & & \\
\hline & $\mathrm{RP}$ & 47.73 & 57.40 & 99.60 & 143.87 & 8.33 & 13.40 & 15.53 & 20.20 & 30.47 & 45.27 & 122.07 & 163.93 & & \\
\hline \multirow{2}{*}{ TO 3} & EP & 30.20 & 43.93 & 104.80 & 169.53 & 5.33 & 8.13 & 10.60 & 11.67 & 28.60 & 88.40 & 111.20 & 144.87 & & \\
\hline & $\mathrm{RP}$ & 28.53 & 44.00 & 110.27 & 148.13 & 9.47 & 14.47 & 11.80 & 13.80 & 29.80 & 45.40 & 125.40 & 171.33 & & \\
\hline \multirow{2}{*}{ TO 4} & EP & 16.73 & 43.33 & 42.87 & 162.87 & 5.27 & 8.20 & 9.60 & 11.60 & 36.07 & 62.40 & 126.93 & 137.13 & & \\
\hline & $\mathrm{RP}$ & 43.73 & 60.53 & 94.27 & 162.07 & 8.40 & 13.80 & 13.33 & 20.13 & 26.33 & 41.87 & 125.80 & 163.13 & & \\
\hline
\end{tabular}

TABLE III

CONVERGENCE SPEED [NB OF OUTER ITERATIONS] TO CONVERGE TO 99.9\% PERFORMANCE FOR NEAR-FAR CO-RT SCENARIO OF FIG. 2 WITH DIFFERENT SETTINGS FOR IPDB. METHOD TWO-TONE RAND IS WITH PERMUTATED TONES ON THE TRANSFORMATION. TONE ORDER (TO) $1=$ [1:K], TONE ORDER $2=$ [K:-1:1], TONE ORDER $3=$ UNIFORM PROBABILITY OF 1 AND 2, TONE ORDER $4=$ RANDOM PERMUATION, EP $=$ CONSTANT EQUAL INIT POWER, RP = RANDOM INIT POWER SATISFYING TOTAL POWER CONSTRAINT. TWO RIGHT MOST COLUMNS CORRESPOND TO ISB PERFORMANCE.

\begin{tabular}{|c|c|c|c|c|c|c|c|c|c|c|c|c|c|c|c|}
\hline \multirow{3}{*}{\multicolumn{2}{|c|}{$\begin{array}{c}\text { Method } \\
\text { Equalization } \\
\text { Granularity }\end{array}$}} & \multicolumn{4}{|c|}{ Two-tone } & \multicolumn{4}{|c|}{ Two-tone rand } & \multicolumn{4}{|c|}{ Three-tone 2} & \multicolumn{2}{|c|}{ ISB } \\
\hline & & \multicolumn{2}{|c|}{ EQ OFF } & \multicolumn{2}{|c|}{ EQ ON } & \multicolumn{2}{|c|}{ EQ OFF } & \multicolumn{2}{|c|}{ EQ ON } & \multicolumn{2}{|c|}{ EQ OFF } & \multicolumn{2}{|c|}{ EQ ON } & \multirow{2}{*}{$\frac{\mathrm{EQ} O F F}{0.5 \mathrm{~dB}}$} & \multirow{2}{*}{$\frac{\text { EQ ON }}{0.5 \mathrm{~dB}}$} \\
\hline & & $1 \mathrm{~dB}$ & $10 \mathrm{~dB}$ & $1 \mathrm{~dB}$ & $10 \mathrm{~dB}$ & $1 \mathrm{~dB}$ & $10 \mathrm{~dB}$ & $1 \mathrm{~dB}$ & $10 \mathrm{~dB}$ & $1 \mathrm{~dB}$ & $10 \mathrm{~dB}$ & $1 \mathrm{~dB}$ & $10 \mathrm{~dB}$ & & \\
\hline \multirow{2}{*}{ TO 1} & EP & 84.00 & 68.00 & 155.00 & 174.00 & 11.07 & 15.53 & 14.00 & 25.13 & 83.00 & 89.00 & 169.00 & 104.00 & 2.00 & 2.00 \\
\hline & $\mathrm{RP}$ & 118.33 & 156.40 & 144.60 & 191.53 & 14.07 & 22.13 & 22.60 & 22.20 & 93.53 & 138.07 & 157.07 & 189.80 & 2.25 & 6.25 \\
\hline \multirow{2}{*}{ TO 2} & EP & 32.00 & 66.00 & 63.00 & 200.00 & 10.60 & 15.13 & 15.87 & 13.87 & 50.00 & 70.00 & 184.00 & 198.00 & & \\
\hline & RP & 125.53 & 137.53 & 124.40 & 168.13 & 13.33 & 20.73 & 21.13 & 27.07 & 82.47 & 127.80 & 163.27 & 189.87 & & \\
\hline \multirow{2}{*}{ TO 3} & $\mathrm{EP}$ & 45.60 & 58.33 & 147.47 & 188.40 & 10.80 & 15.67 & 17.73 & 16.80 & 41.27 & 109.93 & 149.07 & 158.07 & & \\
\hline & RP & 102.67 & 116.13 & 151.73 & 177.67 & 15.13 & 22.80 & 16.93 & 21.60 & 103.73 & 140.27 & 166.87 & 188.27 & & \\
\hline \multirow{2}{*}{ TO 4} & $\mathrm{EP}$ & 59.13 & 67.20 & 63.73 & 177.07 & 11.27 & 16.27 & 14.13 & 17.93 & 42.53 & 82.87 & 138.87 & 164.93 & & \\
\hline & RP & 119.87 & 141.73 & 143.07 & 188.00 & 13.33 & 21.00 & 18.67 & 26.93 & 95.07 & 125.53 & 143.47 & 183.27 & & \\
\hline
\end{tabular}

99\% and $99.9 \%$ accuracy, respectively, as this constitutes the main computational cost of the algorithm. The computational complexity of ISB is taken as a reference. Note that for the dual optimization part in ISB, the dual search is optimized with tuned settings. As IPDB is a primal algorithm, it does not have a dual optimization part, at the cost of more outer iterations. However, it can be seen that IPDB features a much lower overall computational complexity for specific configuration settings. In particular the two-tone rand DoV transformation (15) with $10 \mathrm{dBm} / \mathrm{Hz}$ granularity reduces complexity by a factor 20 (for $99 \%$ performance) and factor 12 (for $99.9 \%$ performance). For IPDB with the two-tone rand
DoV transformation, EQ ON and $1 \mathrm{dBm} / \mathrm{Hz}$ granularity, we observe a 50\% complexity reduction compared to ISB.

A comparison in simulation time strongly depends on the particular implementations of both algorithms. IPDB as well as ISB are implemented in Matlab, without any serious attempt to optimize the implementation of both algorithms. Still, we provide below samples of the observed simulation times for both algorithms, in order to give a more concrete and direct comparison: for IPDB with the two-tone rand DoV transformation we observed a simulation time ranging from 6 seconds up to 77 seconds for $10 \mathrm{dBm} / \mathrm{Hz}$ and $1 \mathrm{dBm} / \mathrm{Hz}$, whereas for ISB we observed simulation times of 15 seconds 


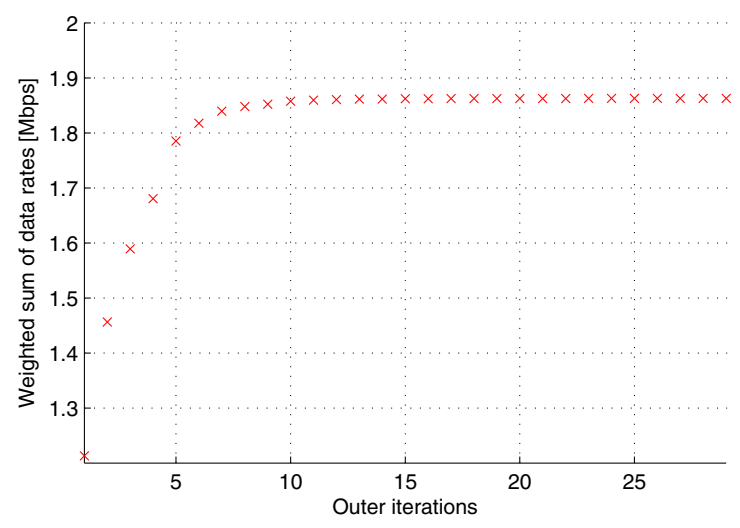

Fig. 3. Evolution of weighted sum of data rates for IPDB algorithm.

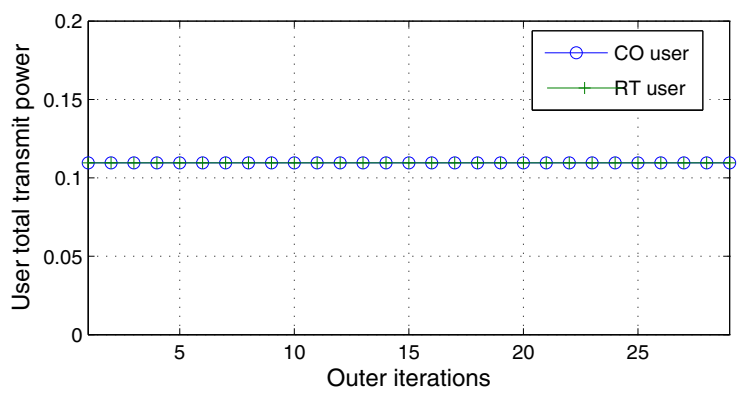

Fig. 4. Evolution of user powers for IPDB algorithm.

up to 150 seconds, depending on the convergence speed of the dual search.

Finally we also compare the computational complexity of IPDB with that of the standard fmincon trust-region and interior-point methods of Matlab. The trust-region method required 400 iterations before convergence to a local optimum, corresponding to 2600 seconds of simulation time and a performance of 1.88769 Mbps. The interior-point method resulted in 648 iterations before convergence to a local optimum, corresponding to 321 seconds of simulation time and a performance of $1.598897 \mathrm{Mbps}$. The IPDB algorithm using the two-tone rand DoV transformation, EQ ON, $1 \mathrm{~dB}$, TO 1 displays thus a better performance (1.8978 Mbps and 1.8951 Mbps), while also being considerably faster.

4) Real-time Property: A main strength of IPDB is its real-time property. To compare with ISB, we determine first the number of power updates that ISB maximally requires to satisfy the per-user total power constraints, taking the inner dual optimization step into account. Compared to one single power difference variable (and thus power) update for IPDB, ISB requires $1.510^{6}$ power updates to satisfy the per-user total power constraints (in the worst-case). This corresponds to the worst-case number of power updates (over all outer iterations) that a user needs to converge to transmit powers so as to satisfy its total power constraint. ISB thus does not qualify as a RT-DSM algorithm, in contrast to IPDB.

5) Equalization Performance: To demonstrate the equalization impact, IPDB is simulated with RP, TO $4,1 \mathrm{dBm} / \mathrm{Hz}$ granularity, two-tone rand DoV transformation and equalization procedure Algorithm 3 activated (EQ ON) for every fifth outer iteration.

The resulting transmit spectra before and after equalization are shown for iteration 5 and 10 in Figure 5. It can be seen that, after the equalization step the transmit spectra display fewer spikes. In this case, all spikes are removed after only two equalization steps, demonstrating the effectiveness of the equalization procedure.

\section{B. Impact of Discretization Granularity: ADSL2+ case}

As mentioned in Section III-C, the discrete grid-based search granularity for the power difference variables for IPDB can be chosen coarser than for the transmit powers for ISB, i.e., $1 \mathrm{dBm} / \mathrm{Hz}$ instead of $0.5 \mathrm{dBm} / \mathrm{Hz}$. In this section we assess the concrete impact of different granularities for an ADSL2+ scenario as given in Figure 6, in terms final transmit spectra. The downstream ADSL2+ scenario consists of 12 users with line lengths $5000 \mathrm{~m}, 4000 \mathrm{~m}, 3000 \mathrm{~m}, 2000 \mathrm{~m}$, $2000 \mathrm{~m}, 1000 \mathrm{~m}, 4800 \mathrm{~m}, 3800 \mathrm{~m}, 2800 \mathrm{~m}, 2300 \mathrm{~m}, 1500 \mathrm{~m}$, and $1300 \mathrm{~m}$. The distances (between CO and RTs) are $0 \mathrm{~m}$, $0 \mathrm{~m}, 1000 \mathrm{~m}, 1000 \mathrm{~m}, 2000 \mathrm{~m}, 2000 \mathrm{~m}, 0 \mathrm{~m}, 0 \mathrm{~m}, 1200 \mathrm{~m}$, $1200 \mathrm{~m}, 2400 \mathrm{~m}$, and $2400 \mathrm{~m}$.

We compare three configurations with each other: a) IPDB with $1 \mathrm{dBm} / \mathrm{Hz}$ granularity, b) IPDB with $10 \mathrm{dBm} / \mathrm{Hz}$ granularity, and c) ISB with $0.5 \mathrm{dBm} / \mathrm{Hz}$ granularity. For the three configurations, we start from the same initial transmit powers (EP setting) and all three converge to similar solutions. The weighted sum achievable data rate performance corresponds to: a) $16.0072 \mathrm{Mbps}$, b) $15.9960 \mathrm{Mbps}$, c) $16.0055 \mathrm{Mbps}$.

In Figure 7, the resulting transmit spectra for user 7 are zoomed out for tones 230 to 420 for the three above configurations. It can be seen that ISB makes steps of $0.5 \mathrm{dBm} / \mathrm{Hz}$. In contrast, the IPDB methods (for both granularities) display a smaller step variation in magnitude, which demonstrates that a much coarser granularity for IPDB does not impact the shape of the resulting transmit spectra too much. Some level of nonsmooth behaviour is seen for IPDB though. The equalization procedure is not able to remove this, because the equalization threshold is set at $10 \mathrm{dBm} / \mathrm{Hz}$ (as shown in lines 3,4,6,7 in Algorithm 3).

\section{Inequality Constraints: VDSL case}

For typical DSL scenarios, all users are allocated their full available transmit power satisfying the total power constraints with equality, i.e., $P^{n}=P^{n \text {,tot }}$. However, for multi-user large crosstalk settings and under specific values of the weights $w_{n}$, it is possible that some users benefit not being allocated all available per-user total power. Here we consider a 6-user VDSL upstream scenario, with 6 CO-connected lines with line lengths $1200 \mathrm{~m}, 1000 \mathrm{~m}, 800 \mathrm{~m}, 600 \mathrm{~m}, 450 \mathrm{~m}$, and $300 \mathrm{~m}$, corresponding to users 1 to 6 , respectively. For this scenario, users 4 and 5 are typically not allocated all available peruser total power, i.e., $P^{n}<P^{n \text {,tot }}$. When running the IPDB algorithm (with RP, EQ ON, $1 \mathrm{dBm} / \mathrm{Hz}$ granularity, TO 4 , DoV transformation (15)) with the inequality procedure of Algorithm 2 (with $\alpha=1.1$ and $\beta=0.8$ ), the evolution of the allocated per-user total powers is shown in Figure 8. It can be seen that the per-user total power constraints are always 
TABLE IV

RELATIVE COMPLEXITY (WRT ISB STANDARD METHOD) TO CONVERGE TO 99\% PERFORMANCE FOR NEAR-FAR CO-RT SCENARIO OF FIG. 2 WITH DIFFERENT SETTINGS FOR IPDB. METHOD TWO-TONE RAND IS WITH PERMUTATED TONES ON THE TRANSFORMATION. TONE ORDER (TO) $1=[1: \mathrm{K}]$, TONE ORDER $2=$ [K:-1:1], TONE ORDER $3=$ UNIFORM PROBABILITY OF 1 AND 2 , TONE ORDER $4=$ RANDOM PERMUATION, EP $=$ CONSTANT EQUAL INIT POWER, RP = RANDOM INIT POWER SATISFYING TOTAL POWER CONSTRAINT. TWO RIGHT MOST COLUMNS CORRESPOND TO ISB PERFORMANCE.

\begin{tabular}{|c|c|c|c|c|c|c|c|c|c|c|c|c|c|c|c|}
\hline \multirow{2}{*}{\multicolumn{2}{|c|}{$\begin{array}{c}\text { Method } \\
\text { Equalization }\end{array}$}} & \multicolumn{4}{|c|}{ Two-tone } & \multicolumn{4}{|c|}{ Two-tone rand } & \multicolumn{4}{|c|}{ Three-tone 2} & \multicolumn{2}{|c|}{ ISB } \\
\hline & & \multicolumn{2}{|c|}{ EQ OFF } & \multicolumn{2}{|c|}{ EQ ON } & \multicolumn{2}{|c|}{ EQ OFF } & \multicolumn{2}{|c|}{ EQ ON } & \multicolumn{2}{|c|}{ EQ OFF } & \multicolumn{2}{|c|}{ EQ ON } & \multirow{2}{*}{$\begin{array}{c}\text { EQ OFF } \\
0.5 \mathrm{~dB}\end{array}$} & \multirow{2}{*}{$\frac{\mathrm{EQ} \mathrm{ON}}{0.5 \mathrm{~dB}}$} \\
\hline \multicolumn{2}{|c|}{ Granularity } & $1 \mathrm{~dB}$ & $10 \mathrm{~dB}$ & $1 \mathrm{~dB}$ & $10 \mathrm{~dB}$ & $1 \mathrm{~dB}$ & $10 \mathrm{~dB}$ & $1 \mathrm{~dB}$ & $10 \mathrm{~dB}$ & $1 \mathrm{~dB}$ & $10 \mathrm{~dB}$ & $1 \mathrm{~dB}$ & $10 \mathrm{~dB}$ & & \\
\hline \multirow{2}{*}{ TO 1} & EP & 2.5164 & 0.2590 & 7.6023 & 1.0543 & 0.2739 & 0.0521 & 0.4999 & 0.1178 & 3.7865 & 0.3494 & 5.1400 & 0.6725 & \multirow[t]{2}{*}{1.00} & \multirow[t]{2}{*}{1.00} \\
\hline & RP & 1.7928 & 0.3406 & 5.6130 & 1.0085 & 0.3594 & 0.0792 & 0.7443 & 0.0878 & 1.4658 & 0.2554 & 6.4844 & 1.0529 & & \\
\hline \multirow{2}{*}{ TO 2} & EP & 1.5272 & 0.3364 & 3.0089 & 1.1248 & \multirow{2}{*}{$\begin{array}{l}0.2723 \\
0.3604\end{array}$} & 0.0521 & \multirow{2}{*}{$\begin{array}{l}0.4949 \\
0.6630\end{array}$} & 0.0521 & \multirow{2}{*}{$\begin{array}{l}1.1267 \\
1.3955\end{array}$} & 0.3675 & \multirow{2}{*}{$\begin{array}{l}5.0723 \\
5.6175\end{array}$} & 1.1433 & & \\
\hline & RP & 2.0256 & 0.3157 & 4.3902 & 0.8718 & & 0.0741 & & 0.1159 & & 0.2603 & & 1.0148 & & \\
\hline \multirow{2}{*}{ TO 3} & EP & 1.6278 & 0.2804 & 5.0953 & 1.1024 & \multirow{2}{*}{$\begin{array}{l}0.2731 \\
0.3991\end{array}$} & 0.0521 & \multirow{2}{*}{$\begin{array}{l}0.4973 \\
0.4947\end{array}$} & 0.0705 & \multirow{2}{*}{$\begin{array}{l}1.4297 \\
1.3478\end{array}$} & 0.5400 & \multirow{2}{*}{$\begin{array}{l}5.0541 \\
5.8502\end{array}$} & 0.9348 & & \\
\hline & $\mathrm{RP}$ & 1.2612 & 0.2516 & 4.9723 & 0.9191 & & 0.0796 & & 0.0765 & & 0.2626 & & 1.0582 & & \\
\hline \multirow{2}{*}{ TO 4} & EP & 0.8795 & 0.2802 & 2.2966 & 1.0672 & 0.2729 & 0.0521 & \multirow{2}{*}{$\begin{array}{l}0.4543 \\
0.5791\end{array}$} & 0.0706 & \multirow{2}{*}{$\begin{array}{l}1.8095 \\
1.2467\end{array}$} & 0.3854 & \multirow{2}{*}{$\begin{array}{l}6.1238 \\
6.2019\end{array}$} & 0.8910 & & \\
\hline & $\mathrm{RP}$ & 1.9321 & 0.3380 & 4.3290 & 1.0169 & 0.3631 & 0.0740 & & 0.1167 & & 0.2398 & & 1.0213 & & \\
\hline
\end{tabular}

TABLE V

RELATIVE COMPLEXITY (WRT ISB STANDARD METHOD) TO CONVERGE TO 99.9\% PERFORMANCE FOR NEAR-FAR CO-RT SCENARIO OF FIG. 2 WITH DIFFERENT SETTINGS FOR IPDB. METHOD TWO-TONE RAND IS WITH PERMUTATED TONES ON THE TRANSFORMATION. TONE ORDER (TO) $1=[1: \mathrm{K}]$, TONE ORDER $2=$ [K:-1:1], TONE ORDER $3=$ UNIFORM PROBABILITY OF 1 AND 2, TONE ORDER $4=$ RANDOM PERMUATION, EP $=$ CONSTANT EQUAL INIT POWER, RP = RANDOM INIT POWER SATISFYING TOTAL POWER CONSTRAINT. TWO RIGHT MOST COLUMNS CORRESPOND TO ISB PERFORMANCE.

\begin{tabular}{|c|c|c|c|c|c|c|c|c|c|c|c|c|c|c|c|}
\hline \multirow{3}{*}{\multicolumn{2}{|c|}{$\begin{array}{c}\text { Method } \\
\text { Equalization } \\
\end{array}$}} & \multicolumn{4}{|c|}{ Two-tone } & \multicolumn{4}{|c|}{ Two-tone rand } & \multicolumn{4}{|c|}{ Three-tone 2} & \multicolumn{2}{|c|}{ ISB } \\
\hline & & \multicolumn{2}{|c|}{ EQ OFF } & \multicolumn{2}{|c|}{ EQ ON } & \multicolumn{2}{|c|}{ EQ OFF } & \multicolumn{2}{|c|}{ EQ ON } & \multicolumn{2}{|c|}{ EQ OFF } & \multicolumn{2}{|c|}{ EQ ON } & \multirow{2}{*}{$\frac{\text { EQ OFF }}{0.5 \mathrm{~dB}}$} & \multirow{2}{*}{$\frac{\mathrm{EQ} \mathrm{ON}}{0.5 \mathrm{~dB}}$} \\
\hline & & $1 \mathrm{~dB}$ & $10 \mathrm{~dB}$ & $1 \mathrm{~dB}$ & $10 \mathrm{~dB}$ & $1 \mathrm{~dB}$ & $10 \mathrm{~dB}$ & $1 \mathrm{~dB}$ & $10 \mathrm{~dB}$ & $1 \mathrm{~dB}$ & $10 \mathrm{~dB}$ & $1 \mathrm{~dB}$ & $10 \mathrm{~dB}$ & & \\
\hline \multirow{2}{*}{ TO 1} & EP & 4.2908 & 0.4292 & 7.8222 & 1.1298 & 0.5398 & 0.0945 & 0.6694 & 0.1513 & 3.9013 & 0.5441 & 7.9446 & 0.6789 & \multirow[t]{2}{*}{1.00} & \multirow[t]{2}{*}{1.00} \\
\hline & $\mathrm{RP}$ & 4.5991 & 0.7979 & 6.4685 & 1.1828 & 0.6071 & 0.1217 & 0.9525 & 0.1274 & 3.7376 & 0.7171 & 7.5192 & 1.1739 & & \\
\hline \multirow{2}{*}{ TO 2} & EP & 1.6558 & 0.4217 & 3.0948 & 1.2865 & \multirow{2}{*}{$\begin{array}{l}0.4924 \\
0.5668\end{array}$} & 0.0945 & \multirow{2}{*}{$\begin{array}{l}0.7036 \\
0.9126\end{array}$} & 0.0826 & \multirow{2}{*}{$\begin{array}{l}2.1677 \\
3.2402\end{array}$} & 0.4326 & \multirow{2}{*}{$\begin{array}{l}7.8462 \\
7.3323\end{array}$} & 1.2738 & & \\
\hline & RP & 4.7169 & 0.7045 & 5.3924 & 1.0159 & & 0.1118 & & 0.1541 & & 0.6662 & & 1.1704 & & \\
\hline \multirow{2}{*}{ TO 3} & EP & 2.3030 & 0.3722 & 6.9218 & 1.2221 & \multirow{2}{*}{$\begin{array}{l}0.4959 \\
0.6448 \\
\end{array}$} & 0.0945 & \multirow{2}{*}{$\begin{array}{l}0.7904 \\
0.7063 \\
\end{array}$} & 0.1003 & 1.9487 & 0.6497 & \multirow{2}{*}{$\begin{array}{l}6.5151 \\
7.5595 \\
\end{array}$} & 1.0230 & & \\
\hline & RP & 3.9985 & 0.6127 & 6.6951 & 1.0937 & & 0.1223 & & 0.1223 & 3.7474 & 0.7235 & & 1.1591 & & \\
\hline \multirow{2}{*}{ TO 4} & EP & 2.8825 & 0.4265 & 3.3138 & 1.1623 & \multirow{2}{*}{$\begin{array}{l}0.5368 \\
0.5702\end{array}$} & 0.1004 & \multirow{2}{*}{$\begin{array}{l}0.6662 \\
0.7883\end{array}$} & 0.1063 & 2.0686 & 0.4900 & \multirow{2}{*}{$\begin{array}{l}6.6522 \\
7.0006\end{array}$} & 1.0611 & & \\
\hline & RP & 4.9687 & 0.7453 & 6.4080 & 1.1770 & & 0.1170 & & 0.1498 & 3.6000 & 0.6725 & & 1.1423 & & \\
\hline
\end{tabular}

satisfied. After 30 iterations, both users achieve their final peruser total power allocation corresponding to $23 \%$ and $1.7 \%$ of full power allocation $P^{n \text {,tot }}$. This demonstrates the real-time property of IPDB while considering inequality constraints.

\section{Downlink Power Control in Heterogeneous Wireless Net- works}

As highlighted in Section I, the proposed RT-DSM theory and IPDB algorithm can also be applied to wireless communication settings. One highly relevant problem is downlink power control in heterogeneous cellullar networks where OFDMA is used within each cell and inter-cell interference (ICIC) occurs between different (macro, pico, femto) cells. In [37] it is explained that this consists of two subproblems, namely a user scheduling part and a power spectrum control part. For the power spectrum control part, it is shown how DSM algorithms can lead to spectacular performance gains. The IPDB algorithm can similarly be applied to this setting so as to obtain real-time inter-cell interference coordination (ICIC) for such heterogeneous networks (HetNet).

For illustration purpose, the IPDB algorithm is applied here to a system with two interfering cells, one macrocell and one femtocell. Each cell has one user. The user in the macrocell is located at the cell edge at a distance of $500 \mathrm{~m}$ from the macrocell base station and $20 \mathrm{~m}$ from the femtocell base station. The user in the femtocell is located at the same location but is served by the femtocell base station. It is known that this constitutes a challenging interference-limited setting. We consider a system bandwidth of $5 \mathrm{MHz}$, a subcarrier spacing of $15 \mathrm{kHz}$, a symbol rate of 14 OFDM symbols in $1 \mathrm{~ms}, 300$ subcarriers, a macrocell base station transmit power of $43 \mathrm{dBm}$ and a femtocell base station transmit power of 15 $\mathrm{dBm}$. The ITU-PED B channel model is used with a pathloss of $31.5+35 \log _{10}$ (distance). The resulting bit loadings are displayed in Figure 9. As the scenario deals with two edge users, the resulting transmit power allocations are OFDMAlike. There is a small overlap though in tones 20-22.

We also compare in Figure 10 the achievable rate region of IPDB with other existing power spectrum control schemes for the considered HetNet scenario. Here, we consider (1) iterative waterfilling (IWF) [38], (2) plain equal power allocation (EP), and (3) time division multiple access (TDMA) where cells are allocated separate time slots with single-cell waterfilling optimized transmit spectra. In addition we evaluate the rate regions of IPDB when only every fourth $(4 \mathrm{x})$ and eighth $(8 \mathrm{x})$ tone is considered, and interpolating in between. This roughly reduces complexity by a factor of 4 and 8 , respectively. It can be seen that IPDB results in a good network performance tradeoff, even for the reduced complexity IPDB solutions (IPDB $(4 x)$ and IPDB (8x)). We want to emphasize however that the considered HetNet scenario is rather simple and one cannot make a general statement on the performance of IPDB for general HetNet scenarios. A more rigorous assessment would require an extensive performance evaluation using realistic and 

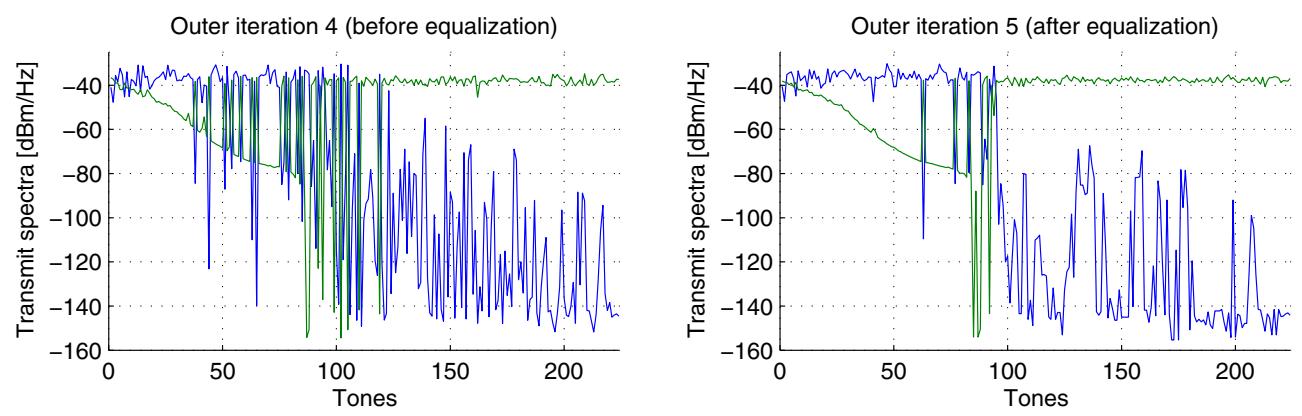

Outer iteration 9 (before equalization)
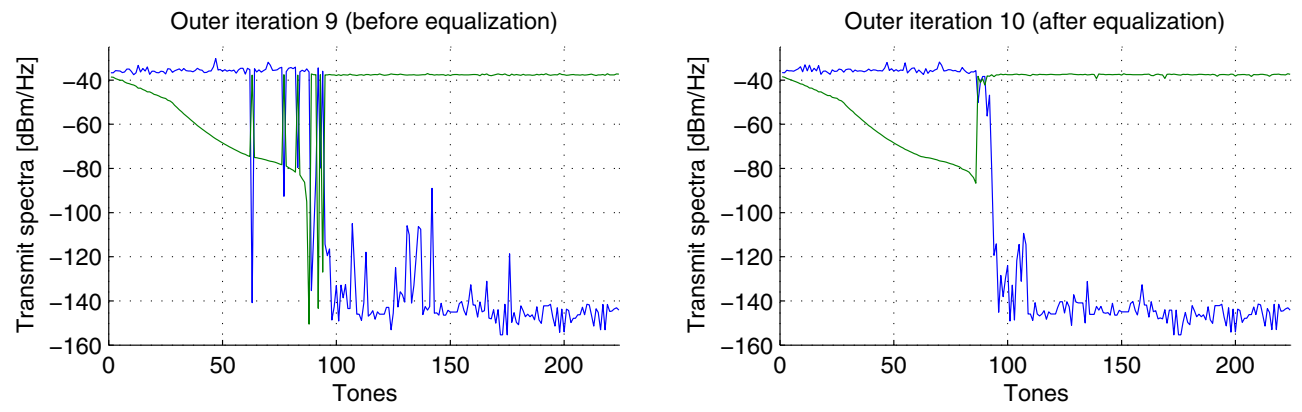

Fig. 5. Impact when equalization procedure Algorithm 3 is activated for IPDB at outer iteration 5 and 10, before and after the equalizations. Transmit spectra of CO-user and RT-user in Figure 2 are displayed in blue and green, respectively.

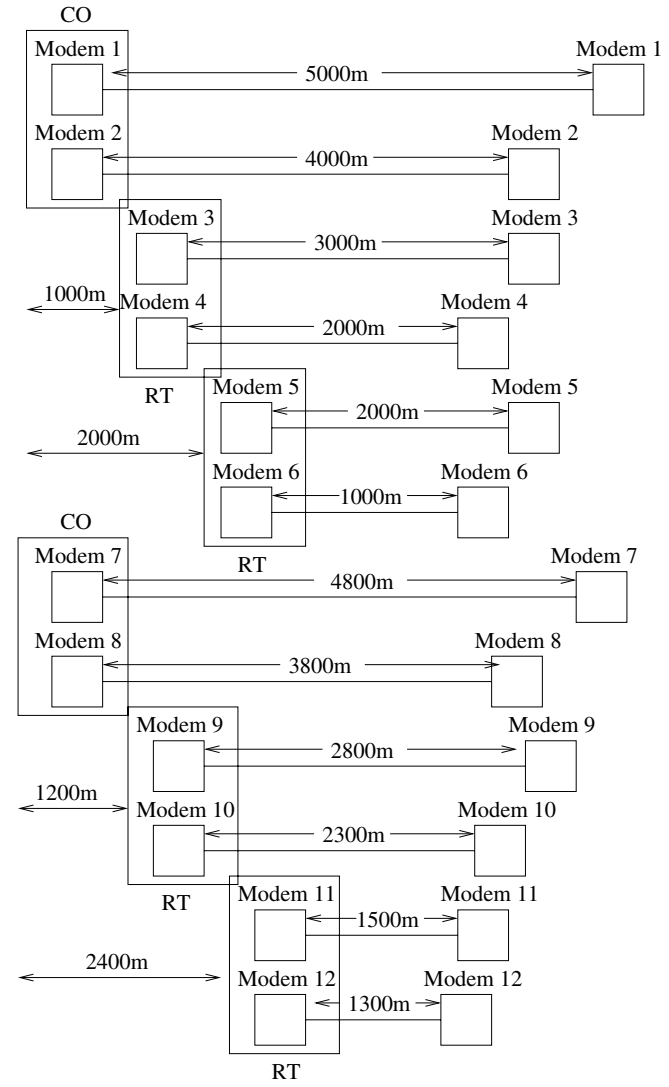

Fig. 6. ADSL2+ scenario.

practical network types and channel measurements. The goal of this section is however not to analyze the resulting transmit spectra, bit loadings and performance for realistic systems, but to demonstrate the wide applicability of the proposed RT-DSM theory and algorithm beyond the wireline DSL setting.

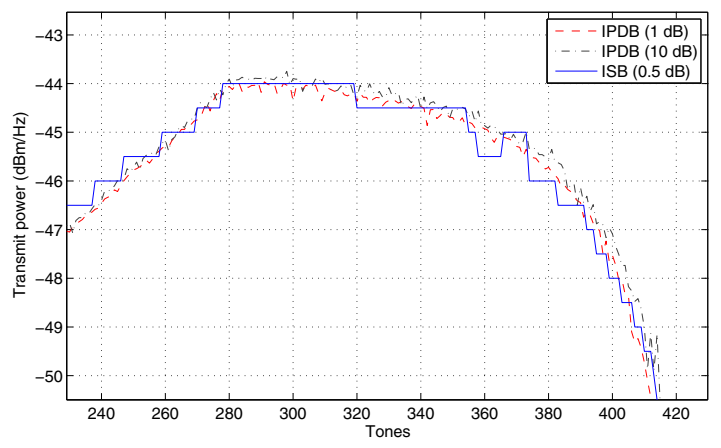

Fig. 7. Zoom of transmit spectra for user 7 of 12-user ADSL2+ scenario.

\section{CONClusion}

We have proposed a new paradigm and theory for RTDSM in multi-user multi-carrier communication systems. A new RT-DSM algorithm, referred to as IPDB, is proposed and is suitable for operation under tight computation time and compute power constraints, i.e., when a very fast responsiveness is required. IPDB can be stopped at any moment in time during execution while guaranteeing feasibility and improved performance. The IPDB algorithm design is enabled by a novel transformation, referred to as the DoV transformation, which transforms the standard DSM problem into an alternative but equivalent optimization problem with primal power difference variables. A coordinate ascent approach is proposed to tackle the reformulated primal problem with an iterative 1D exact line search via a logarithmically-scaled grid search. In contrast to existing DSM algorithms that follow a dual decomposition approach, IPDB solves the DSM problem in the primal domain, avoiding any potential issues with a non-zero duality gap, which can be seen as an important 


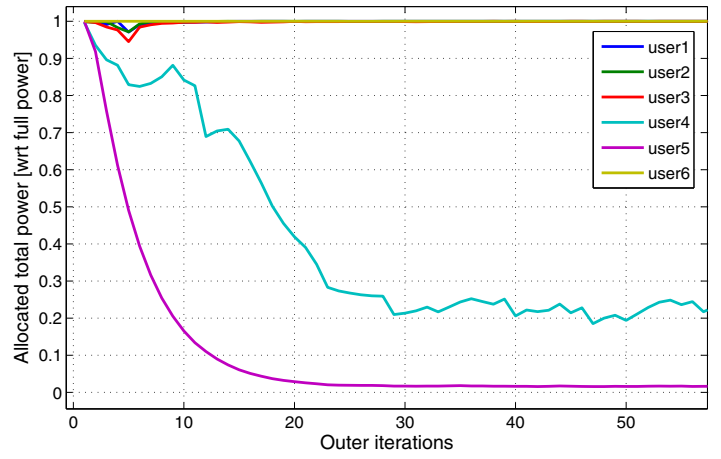

Fig. 8. Evolution of user powers for IPDB with inequality procedure of Algorithm 2 for 6-user upstream VDSL scenario.

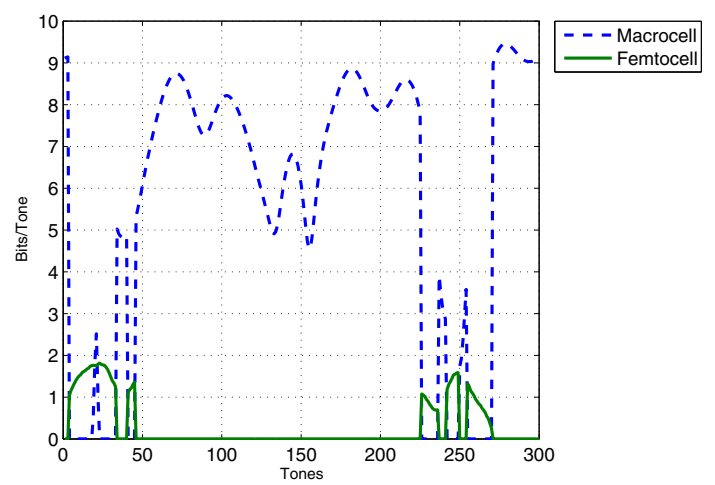

Fig. 9. Bit allocation with IPDB for LTE heterogeneous network with macroand femtocell inter-cell interference.

benefit. IPDB is furthermore characterized by a high tunability with additional procedures for dealing with inequalities and equalization that result in improved performance and smoother transmit power spectra. In particular, the configuration with the 'two-tone rand' DoV transformation and equalization results in fast convergence, good network-wide achievable data rate performance, low computational cost, and real-time execution, outperforming the existing near-optimal ISB algorithm. This has been validated with simulations under different configuration settings for different practical wireline XDSL scenarios and for a wireless LTE heterogeneous network scenario.

\section{REFERENCES}

[1] D. Gesbert, S. Hanly, H. Huang, S. S. Shitz, O. Simeone, and W. Yu, "Multi-cell MIMO cooperative networks: a new look at interference," IEEE J. Sel. Areas Commun., vol. 28, no. 9, pp. 1380-1408, Dec. 2010.

[2] J. Lee, Y. Kim, H. Lee, B. L. Ng, D. Mazzarese, J. Liu, W. Xiao, and Y. Zhou, "Coordinated multipoint transmission and reception in LTEadvanced systems," IEEE Commun. Mag., vol. 50, no. 11, pp. 44-50, 2012.

[3] K. Zheng, F. Hu, W. Wang, W. Xiang, and M. Dohler, "Radio resource allocation in LTE-advanced cellular networks with M2M communications," IEEE Commun. Mag., vol. 50, no. 7, pp. 184-192, 2012.

[4] D. Lee, H. Seo, B. Clerckx, E. Hardouin, D. Mazzarese, S. Nagata, and K. Sayana, "Coordinated multipoint transmission and reception in LTE-advanced: deployment scenarios and operational challenges," IEEE Commun. Mag., vol. 50, no. 2, pp. 148-155, 2012.

[5] K. Song, S. Chung, G. Ginis, and J. M. Cioffi, "Dynamic spectrum management for next-generation DSL systems," IEEE Commun. Mag., vol. 40, no. 10, pp. 101-109, Oct. 2002.

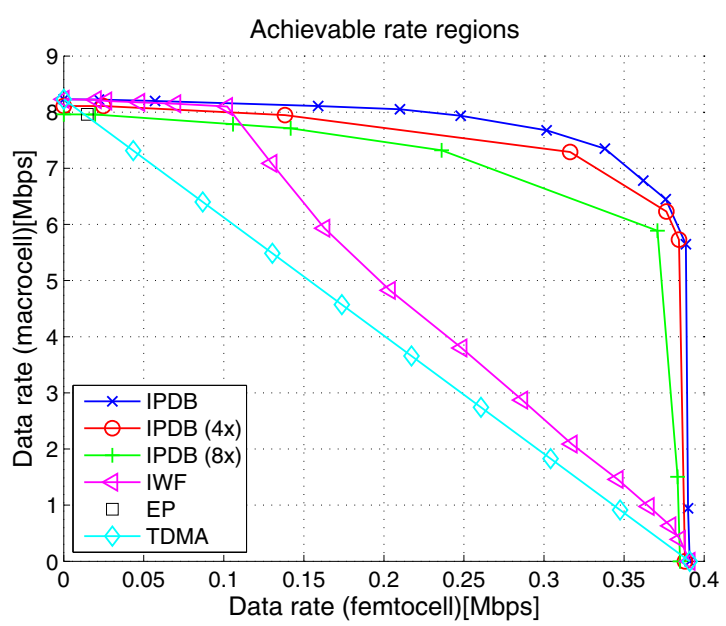

Fig. 10. Rate Regions with ICIC power spectrum control in HetNet scenario.

[6] G. Ginis and J. M. Cioffi, "Vectored transmission for digital subscriber line systems," IEEE J. Sel. Areas Commun., vol. 20, no. 5, pp. 10851104, Jun. 2002.

[7] R. Cendrillon, "Multi-user signal and spectra co-ordination for digital subscriber lines," Ph.D. dissertation, KU Leuven, Belgium, Dec. 2004.

[8] "Dynamic line management for vectoring scenarios," Alcatel-Lucent, Strategic White Paper, 2013. Available: resources.alcatel $\backslash$-lucent.com/ ?cid=167693 \\&REFFER=alu.prod.detail $\backslash \% 20 \backslash \% 7 \mathrm{C} \backslash \% 20$ Motive $\backslash \backslash \backslash$ $\% 20$ Network $\backslash \% 20$ Analyzer $\backslash \% 20-\backslash \% 20$ Copper

[9] J. Mao, G. Xie, J. Gao, and Y. Liu, "Energy efficiency optimization for OFDM-based cognitive radio systems: a water-filling factor aided search method," IEEE Trans. Wireless Commun., vol. 12, no. 5, pp. 2366-2375, 2013.

[10] M. Wolkerstorfer, D. Statovci, and T. Nordström, "Dynamic spectrum management for energy-efficient transmission in DSL," in Proc. 2008 IEEE International Conference on Communications Systems.

[11] P. Tsiaflakis, Y. Yi, M. Chiang, and M. Moonen, "Green DSL: energyefficient DSM," in Proc. 2009 IEEE International Conference on Communications.

[12] J. M. Cioffi, S. Jagannathan, W. Lee, H. Zou, A. Chowdhery, W. Rhee, G. Ginis, and P. Silverman, "Greener copper with dynamic spectrum management," in 2008 IEEE Global Telecommunications Conference.

[13] K. Hooghe and M. Guenach, "Toward green copper broadband access networks," IEEE Commun. Mag., vol. 49, no. 8, pp. 87-93, Aug. 2011.

[14] M. Monteiro, N. Lindqvist, and A. Klautau, "Spectrum balancing algorithms for power minimization in DSL networks," in Proc. 2009 IEEE International Conference on Communications, pp. 1-5.

[15] M. Wolkerstorfer, S. Trautmann, T. Nordström, and B. D. Putra, "Modeling and optimization of the line-driver power consumption in xDSL systems," EURASIP J. Advances in Signal Process., vol. 2012, no. 226, pp. 1-15, 2012.

[16] S. Panigrahi, Y. Xu, and T. Le-Ngoc, "Multiuser margin optimization in digital subscriber line (DSL) channels," IEEE J. Sel. Areas Commun., vol. 24, no. 8, pp. 1571-1580, Aug. 2006.

[17] S. Jagannathan, C. S. Hwang, and J. M. Cioffi, "Margin optimization in digital subscriber lines employing level-2 dynamic spectrum management," in Proc. 2008 IEEE International Conference on Communications, pp. 435-440.

[18] W. Sarhan, A. Klein, and M. Kuipers, "Jointly optimizing the virtual noise mask and the SNR margin for improved service in xDSL systems," in Proc. 2012 IEEE International Conference on Communications, pp. 3590-3594.

[19] G. Ginis, "Low-power modes for ADSL2 and ADSL2+," Broadband Communications Group, Texas Instruments, Tech. Rep., Jan. 2005.

[20] P. Tsiaflakis, Y. Yi, M. Chiang, and M. Moonen, "Fair greening of broadband access: spectrum management for energy-efficient DSL networks," EURASIP J. Wireless Commun. and Networking, vol. 2011:140, 2011.

[21] P. Tsiaflakis, M. Diehl, and M. Moonen, "Distributed spectrum management algorithms for multiuser DSL networks," IEEE Trans. Signal Process., vol. 56, no. 10, pp. 4825-4843, Oct. 2008.

[22] D. Statovci, T. Nordström, and R. Nilsson, "The normalized-rate iterative algorithm: A practical dynamic spectrum management method for 
DSL," EURASIP J. Appl. Signal Process., vol. 2006, no. ID 95175, pp. $1-17,2006$.

[23] A. Forouzan, M. Moonen, J. Maes, and M. Guenach, "Joint level 2 and 3 dynamic spectrum management for downstream DSL," IEEE Trans. Commun., vol. 60, no. 10, pp. 3111-3122, Oct. 2012.

[24] R. B. Moraes, P. Tsiaflakis, J. Maes, and M. Moonen, "DMT MIMO IC rate maximization in DSL with combined signal and spectrum coordination," IEEE Trans. Signal Process., vol. 61, no. 7, pp. 17561769, Apr. 2013.

[25] C. Leung, S. Huberman, K. Ho-Van, and T. Le-Ngoc, "Vectored DSL: potential, implementation issues and challenges," IEEE Commun. Surveys \& Tutorials, vol. PP, no. 99, pp. 1-17, Jan. 2013.

[26] S. Huberman, C. Leung, and T. Le-Ngoc, "Dynamic spectrum management (DSM) algorithms for multi-user xDSL," IEEE Commun. Surveys and Tutorials, vol. 14, no. 1, First Quarter 2012.

[27] T. Wang and L. Vandendorpe, "Successive convex approximation based methods for dynamic spectrum management," in Proc. 2012 IEEE International Conference on Communications, pp. 4061-4065.

[28] W. Yu and R. Lui, "Dual methods for nonconvex spectrum optimization of multicarrier systems," IEEE Trans. Commun., vol. 54, no. 7, Jul. 2006.

[29] Z. Q. Luo and S. Zhang, "Dynamic spectrum management: complexity and duality," IEEE J. Sel. Topics Signal Process., vol. 2, no. 1, pp. 57-73, Feb. 2008.

[30] R. Cendrillon, W. Yu, M. Moonen, J. Verlinden, and T. Bostoen, "Optimal multiuser spectrum balancing for digital subscriber lines," IEEE Trans. Commun., vol. 54, no. 5, pp. 922-933, May 2006.

[31] R. Cendrillon and M. Moonen, "Iterative spectrum balancing for digital subscriber lines," in Proc. 2005 IEEE International Conference on Communications, vol. 3, pp. 1937-1941.

[32] R. Lui and W. Yu, "Low-complexity near-optimal spectrum balancing for digital subscriber lines," in Proc. 2005 IEEE International Conference on Communications, vol. 3, pp. 1947-1951.

[33] P. Tsiaflakis, J. Vangorp, M. Moonen, and J. Verlinden, "A low complexity optimal spectrum balancing algorithm for digital subscriber lines," Signal Process., vol. 87, no. 7, pp. 1735-1753, Jul. 2007.

[34] P. Weeraddana, M. Codreanu, M. Latva-aho, and A. Ephremides, "Weighted sum-rate maximization for a set of interfering links via branch and bound," IEEE Trans. Signal Process., vol. 59, no. 8, pp. 3977-3996, Aug. 2011.

[35] M. Wolkerstorfer, J. Jalden, and T. Nordström, "Column generation for discrete-rate multi-user and multi-carrier power control," IEEE Trans. on Commun., vol. 60, no. 9, pp. 2712-2722, Sep. 2012.

[36] R. Cendrillon, J. Huang, M. Chiang, and M. Moonen, "Autonomous spectrum balancing for digital subscriber lines," IEEE Trans. Signal Process., vol. 55, no. 8, pp. 4241-4257, Aug. 2007.

[37] K. Son, S. Lee, Y. Yi, and S. Chong, "REFIM: a practical interference management in heterogeneous wireless access networks," IEEE J. Sel. Areas Commun., vol. 29, no. 6, pp. 1260-1272, Jun. 2011.

[38] W. Yu, G. Ginis, and J. M. Cioffi, "Distributed multiuser power control for digital subscriber lines," IEEE J. Sel. Areas Commun., vol. 20, no. 5, pp. 1105-1115, Jun. 2002.

[39] S. Huberman, C. Leung, and T. Le-Ngoc, "Constant offset autonomous spectrum balancing using multiple reference lines for VDSL," IEEE Trans. Signal Process., vol. 60, no. 12, pp. 6719-6723, Dec. 2012.

[40] Z.-Q. Luo and J.-S. Pang, "Analysis of iterative waterfilling algorithm for multiuser power control in digital subscriber lines," EURASIP $J$. Advances Signal Process., vol. 2006, no. 024012, Mar. 2006.

[41] J. Papandriopoulos and J. S. Evans, "SCALE: a low-complexity distributed protocol for spectrum balancing in multiuser DSL networks," IEEE Trans. Inf. Theory, vol. 55, no. 8, pp. 3711-3724, Aug. 2009.

[42] W. Yu, "Multiuser water-filling in the presence of crosstalk," in 2007 Information Theory and Applications Workshop.

[43] Scholarpedia - digital subscriber line. Available: http://www. scholarpedia.org/article/Digital $\backslash \_$subscriber $\backslash$ line $\backslash$ (DSL)

[44] S. Ren and M. van der Schaar, "Distributed power allocation in multi-user multi-channel cellular relay networks," IEEE Trans. Wireless Commun., vol. 9, no. 6, pp. 1952-1964, Jun. 2010.

[45] M. Chiang, P. Hande, T. Lan, and C. Tan, "Power control in wireless cellular networks," Foundations and Trends in Networking, vol. 2, no. 4, pp. 381-533, 2008.

[46] G. Bansal, J. Hossain, and V. K. Bhargava, "Optimal and suboptimal power allocation schemes for OFDM-based cognitive radio systems," IEEE Trans. Wireless Commun., vol. 7, no. 11, pp. 4710-4718, 2008.

[47] T. Starr, J. M. Cioffi, and P. J. Silverman, Understanding Digital Subscriber Lines. Prentice Hall, 1999.
[48] P. Tsiaflakis, Y. Yi, M. Chiang, and M. Moonen, "Throughput and delay performance of DSL broadband access with cross-layer dynamic spectrum management," IEEE Trans. Commun., vol. 60, no. 9, pp. 2700 2711, Sep. 2012.

[49] J. Nocedal and S. J. Wright, Numerical Optimization, 2nd ed. Springer, 2006.

[50] D. P. Bertsekas, Nonlinear Programming, 2nd ed. Athena Scientific, 1999.

[51] Y. Nesterov, "Efficiency of coordinate descent methods on huge-scale optimization problems," SIAM J. Optimization, vol. 22, no. 2, pp. 341362,2012

[52] Asymmetric digital subscriber line (ADSL) transceivers Extended bandwidth ADSL2 (ADSL2+), ITU-T Std. G.992.5, 2005.

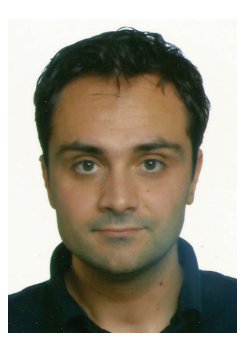

Paschalis Tsiaflakis (S'06-M'09) received the Masters degree in electrical engineering and the Ph.D. degree in engineering sciences from the KU Leuven (Belgium) in 2004 and 2009, respectively, after which he hold a postdoctoral research fellow position from 2010 until 2013. He was a visiting researcher at Princeton University in 2007, a visiting postdoc at the University of California Los Angeles in 2010, and a postdoctoral research associate at the Center for Operations Research and Econometrics in 2011. He is currently a researcher at Bell Labs Alcatel-Lucent. His research expertise is centered around signal processing and optimization for wireline and wireless communication systems.

Dr. Tsiaflakis received the "Belgian Young ICT Personality" award sponsored by FITCE in 2010, two IEEE ICC best paper awards in 2013, the "Best Multimedia Master Thesis" prize award sponsored by PIMC in 2001, and was a top-12 finalist for the European ERCIM Cor Baayen Award 2010. He also received a FWO Aspirant Grant in 2004, a PDMK postdoc grant in 2009, a Francqui Intercommunity Postdoc Grant in 2010, a FWO postdoc grant in 2011, and a FNRS postdoc grant in 2011.

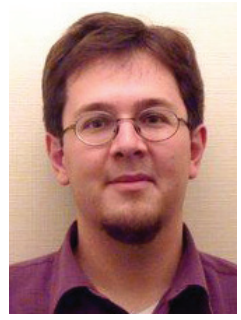

François Glineur received engineering degrees from Faculté Polytechnique de Mons (Belgium) and École supérieure d'électricité (France) in 1997, and a Ph.D. in applied sciences from Faculté Polytechnique de Mons in 2001. He is currently professor of applied mathematics at the Engineering School of Université catholique de Louvain, member of the Center for Operations Research and Econometrics and the Institute of Information and Communication Technologies, Electronics and Applied Mathematics. His main research interests lie in optimization models and algorithms (with a focus on convex optimization) and their engineering applications, as well as in nonnegative matrix factorization.

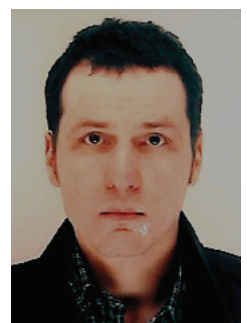

Marc Moonen (M'94-SM'06-F'07) is a Full Professor at the Electrical Engineering Department of $\mathrm{KU}$ Leuven, where he is heading a research team working in the area of numerical algorithms and signal processing for digital communications, wireless communications, DSL and audio signal processing.

He received the 1994 KU Leuven Research Council Award, the 1997 Alcatel Bell (Belgium) Award (with Piet Vandaele), the 2004 Alcatel Bell (Belgium) Award (with Raphael Cendrillon), and was a 1997 Laureate of the Belgium Royal Academy of Science. He received a journal best paper award from the IEEE Transactions on Signal Processing (with Geert Leus) and from Elsevier Signal Processing (with Simon Doclo). He was chairman of the IEEE Benelux Signal Processing Chapter (1998-2002), a member of the IEEE Signal Processing Society Technical Committee on Signal Processing for Communications, and President of EURASIP (European Association for Signal Processing, 2007-2008 and 2011-2012).

He has served as Editor-in-Chief for the EURASIP Journal on Applied Signal Processing (2003-2005), and has been a member of the editorial board of IEEE Transactions on Circuits ANd Systems II, IEEE Signal Processing Magazine, Integration-the VLSI Journal, EURASIP Journal on Wireless Communications and Networking, and Signal Processing. He is currently a member of the editorial board of EURASIP Journal on Applied Signal Processing and Area Editor for Feature Articles in IEEE Signal Processing Magazine. 